Full Paper

\title{
Herkunftsnachweis von Südtiroler Äpfeln mittels Analyse des Sr- Isotopenverhältnisses: Grundlagen und praktische Anwendungen
}

\author{
Determination of origin of South Tyrolean apples using Sr isotope ratio analysis: fundamentals and \\ implementation
}
Determinazione dell'origine delle mele alto atesine mediante l'analisi del rapporto isotopico dello stronzio: principi ed applicazione

\author{
Samira Chizzali ${ }^{1}$, Michele Bassi ${ }^{1}$, Emanuela Pignotti ${ }^{1}$, Agnese Aguzzoni ${ }^{2}$, Francesca Scandellari², Giulio Voto ${ }^{3}$, Pietro Zignale ${ }^{3}$, Walter Guerra ${ }^{1}$, \\ Massimo Tagliavini ${ }^{2}$, Werner Tirler $^{3}$, Peter Robatscher ${ }^{1}$ \\ 1 Versuchszentrum Laimburg, Pfatten, Italien \\ 2 Fakultät für Naturwissenschaften und Technik, Freie Universität Bozen, Bozen, Italien \\ ${ }^{3}$ Eco Research $\mathrm{GmbH}$, Bozen, Italien
}

\section{ABSTRACT}

The origin of a food product is becoming increasingly important for consumers. One promising technique to prove the origin of agricultural products is the analysis of the strontium $(87 \mathrm{Sr} / 86 \mathrm{Sr}$ ) isotope ratio. In crops, this ratio is generally related to the soilgeological features of the corresponding growing area. The present contribution summarizes some aspects of the $87 \mathrm{Sr} / 86 \mathrm{Sr}$ ratio and its possible use as a geographical tracer for apples, investigated during a 3-year project. In a first experiment, we investigated the influence of agricultural practices and their external addition of strontium on the isotope ratio of apple trees under greenhouse conditions. The trees adapted slowly their $87 \mathrm{Sr} / 86 \mathrm{Sr}$ ratio to that of the soil. The addition of tap water and fertilizers modestly influenced the $87 \mathrm{Sr} / 86 \mathrm{Sr}$ ratio of the trees. In a second experiment, we investigated the $87 \mathrm{Sr} / 86 \mathrm{Sr}$ ratio variability in terms of intra-part (difference between sub-samples of the same plant organ within a single tree), intra-tree (difference among the plant organs of the same tree) and inter-tree (differences between each plant-organ of different trees in the same orchard) variabilities and its relationship with the soil isotope ratio. The study was conducted in two orchards in South Tyrol. The intra-part and intra-tree $87 \mathrm{Sr} / 86 \mathrm{Sr}$ ratio in both orchards showed a homogeneous distribution, but inter-tree we observed significant differences between the two orchards. As a third experiment, we extended our analysis to 41 orchards, distributed in North Italy. The $87 \mathrm{Sr} / 86 \mathrm{Sr}$ ratio of apples with geographical indication were compared with those from non-protected districts. In both cases, the isotope ratio of the apples was highly correlated with the soil ratio of the corresponding orchard. However, areas having similar geological features were also characterized by similar ratios, and so a clear separation of all areas was not possible. This confirms the potential of the $87 \mathrm{Sr} / 86 \mathrm{Sr}$ ratio as geographical tracer, but future studies should include other parameters, to improve the classification rate of agricultural products according to their place of origin.

\section{Keywords}

food traceability, strontium isotopes, geographical origin, malus $x$ domestica, MC-ICP-MS

\section{CITE ARTICLE AS}

Chizzali Samira, Bassi Michele, Pignotti Emanuela et.al. (2020). Determination of origin of South Tyrolean apples using $\mathrm{Sr}$ isotope ratio analysis: fundamentals and implementation. Laimburg Journal 02/2020

DOI: $10.23796 /$ L/2020.010

\section{CORRESPONDING AUTHOR}

Peter Robatscher

Versuchszentrum Laimburg, Laimburg 6, Pfatten, 39040 Auer (BZ), Italy

peter.robatscher@laimburg.it +390471414842 


\section{EINLEITUNG}

Der geographische Ursprung der konsumierten Lebensmittel rückt immer mehr in den Fokus der Verbraucher. Aus einer Umfrage der Generaldirektion Landwirtschaft und ländliche Entwicklung der Europäischen Union geht hervor, dass die Herkunft einen erheblichen Einfluss auf die Kaufentscheidung eines Produktes für den Konsumenten hat [1]. Viele Kunden verspüren den Wunsch, den lokalen Markt zu unterstützen, aber auch Umweltaspekte (v.a. hinsichtlich des CO2-Fingerabdrucks) spielen eine Rolle, welche das Interesse an Lebensmittel mit lokaler Herkunft steigern [2] [3]. Geographische Ursprungsbezeichnungen helfen Landwirten und Produzenten, ihre Erzeugnisse zu kennzeichnen und die Verbindung zu einer bestimmten Region herzustellen [4]. In den meisten Fällen wird für Produkte mit geographischer Ursprungsbezeichnung ein höherer Preis erzielt [5]. Aus diesem Grund kann die Kennzeichnung auch missbräuchlich eingesetzt werden, um einen höheren Verkaufspreis und größeren Marktanteil zu erzielen. Das Vorkommen solcher fälschlich gekennzeichneten Produkte sollte zunehmends - auch im Sinne der Produzenten überwacht werden, um die Verbraucher, welche bereit sind, für lokale Produkte höhere Ausgaben zu tätigen, vor Täuschung zu schützen. Dafür sind zuverlässige analytische Nachweismethoden notwendig, insbesondere um juristische Fälle wie Klagen von Verbrauchern und Produzenten mit rechtlich zulässigen Beweisen zu unterstützen [6] und auch Produzenten vor fälschlich deklarierter Ware zu schützen. Unterschiedlichste analytischen Methoden kommen dafür bereits zur Anwendung, wie die Nah-Infrarot Spektroskopie (NIRS), elektronische Nasen gekoppelt mit Massenspektrometrie (MS), Flüssigchromatographie, Fluoreszenzspektroskopie, Kapillarelektrophorese, KernMagnetresonanz (NMR) und die DNA-Analyse [7] [8] [9]. Eine Methode, welche in den letzten Jahren immer häufiger angewandt wurde, ist die Isotopenanalyse, insbesondere der leichten Elemente Kohlenstoff, Wasserstoff, Sauerstoff und Stickstoff, aber auch schwerer wie Strontium oder Blei [9]. Das Element Strontium besitzt vier natürlich vorkommende Isotope: drei davon treten in einer stetig konstanten Menge auf (84Sr, $86 \mathrm{Sr}, 88 \mathrm{Sr}$ ) wohingegen das vierte Isotop $87 \mathrm{Sr}$ durch natürlichen radioaktiven Zerfall aus 87Rb (Halbwertszeit 48800000000 Jahre) entsteht und sich somit seine Anzahl über die Jahrhunderte hinweg langsam erhöht [10]. Die Sr-Isotopenzusammensetzung in Mineralen hängt von ihrem Alter und ihrem initialen $\mathrm{Rb} / \mathrm{Sr}$-Verhältnis ab. Älteres Gestein besitzt meist einen höheren Gehalt an 87Sr im Vergleich zu jüngerem Gestein, sofern beide über dasselbe initiale $\mathrm{Rb} / \mathrm{Sr}$ Verhältnis verfügen. Gestein aus derselben Gesteinsformation mit einem höherem $\mathrm{Rb} / \mathrm{Sr}$-Verhältnis erreicht höhere $87 \mathrm{Sr} / 86 \mathrm{Sr}$ Verhältnisse als jenes mit einem geringeren $\mathrm{Rb} / \mathrm{Sr}$-Verhältnis. Aus diesem Grund hängt das $87 \mathrm{Sr} / 86 \mathrm{Sr}$-Verhältnis im Gestein stark von den lokalen geomorphologischen Verhältnissen ab und reicht von $87 \mathrm{Sr} / 86 \mathrm{Sr}$-Verhältnissen von 0,703 im Erdmantel bis zu > 0,900 im Granit des Archäikums und Metasedimenten der alten kontinentalen Kruste [10] [11] [12]

Durch Verwitterungsprozesse des Grundgesteins geben Minerale Strontium an den Boden ab. Da jedes Mineral ein spezifisches 87Sr/86Sr-Verhältnis und eine eigene $\mathrm{Sr}$ Verwitterungsrate hat, entspricht das Sr-Isotopenverhältnis des Bodens einem Durchschnittswert des zugrundeliegenden Grundgesteins [15] [16]. Der Verwitterungsprozess des Grundgesteins stellt die Hauptquelle an Strontium für den Boden dar. Es gibt allerdings noch weitere Quellen, wodurch es in den Boden gelangen kann (Abb. 1). Dazu gehören unterschiedlichste Wetterbedingungen wie trockene Winde oder Regen,

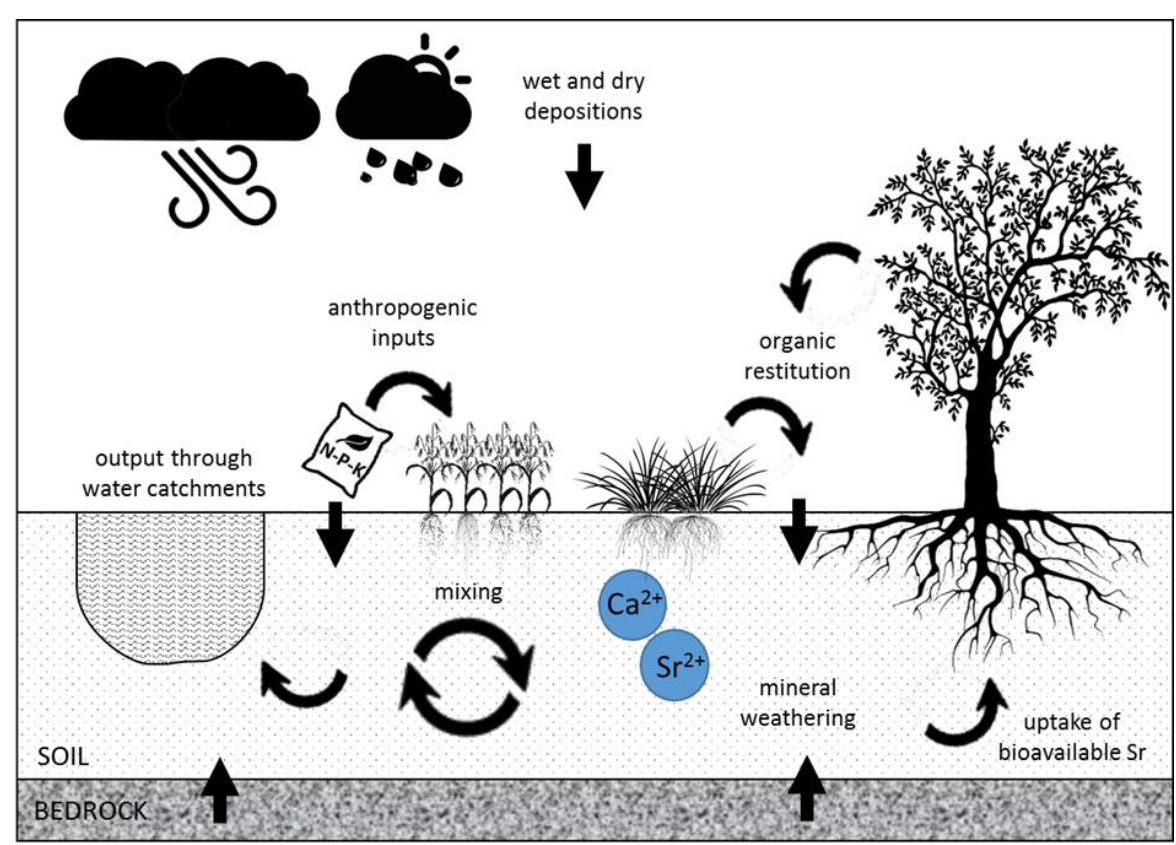

Abb. 1: Schema des Strontium-Zyklus // Scheme of the strontium cycle. Source:[14], p. 16 anthropogene Quellen, wie das Einsetzen von Düngern oder das Einbringen von organischem Material durch z.B. Pflanzen. Strontium kann allerdings auch durch unterschiedlichste Vorgänge wieder aus dem Boden herausgelöst werden, wie z.B. durch das Oberflächenwasser und Grundwasser [13]. Obwohl Strontium nicht zu den essenziellen Elementen gehört, kann es trotzdem von den Wurzeln über den gleichen Weg absorbiert werden wie Calcium (Ca). Die beiden Elemente ( $\mathrm{Ca}$ und $\mathrm{Sr}$ ) verfügen über einige Gemeinsamkeiten wie denselben lonenradius und Valenz, und können aufgrund dessen von der Pflanze nicht unterschieden werden, weshalb sie beide von der Pflanze aufgenommen werden. Für jede Pflanze kann ein Boden-zu-Pflanze-Übertragungsfaktor ermittelt werden, um die Aufnahmekapazitäten der beiden Elemente ( $\mathrm{Sr}$ und $\mathrm{Ca}$ ) zu vergleichen. Dieser Faktor entspricht dem Verhältnis aus der Konzentration des jeweiligen Elementes in der Pflanze und der Konzentration im Boden [17] [18] [19]. Da die verschiedenen $\mathrm{Sr}$-Isotope eine sehr geringe Massendifferenz aufweisen unterliegt $\mathrm{Sr}$ nur einer unbedeutenden Massenfraktionierung (= Unterschiedlich hohe Aufnahme der verschiedenen Isotope in die Pflanze, hervorgerufen durch physikalische oder chemische Prozesse), welche durch natürliche oder instrumentale Prozesse ausgelöst werden kann. Diese auftretende Fraktionierung 
kann mathematisch korrigiert werden, um das Ergebnis der Isotopenverhältnisanalyse der Pflanze nicht zu beeinflussen [20] [21]. Aufgrund dieser Korrektur sollte das 87Sr/86Sr-Isotopenverhältnis in der Pflanze dem des Bodens, auf dem sie wächst, entsprechen [22] [23] [24]. Es kann somit wie der „Fingerabdruck" einer Pflanze gesehen werden, welcher von ihrer geologischen Umgebung abhängig ist und somit eine Möglichkeit zur Nachverfolgung ihrer Herkunft darstellt. Das 87Sr/86Sr-Verhältnis in der Pflanze und im Boden kann durch unterschiedlichste Faktoren beeinflusst werden (Abb. 1). Besonders in der Landwirtschaft könnte dieses Verhältnis durch den Einsatz von Düngern, der Bewässerung und auf die Pflanze gesprühte Phytochemikalien oder Nährstoffe, wodurch $\mathrm{Sr}$ von außen eingebracht werden kann, verändert werden. Diese Vorgänge könnten die Isotopenzusammensetzung hypothetisch nachhaltig verändern, wodurch die eindeutig zuordenbare Verbindung zum Ursprungsgebiet (Boden) nicht mehr möglich wäre [25] [26] [27]. Böden können geologisch sehr komplex zusammengesetzt sein. Dadurch, dass Minerale oft ungleichmäßig vertikal und/oder horizontal im Boden verteilt sind, auch durch menschliche oder mechanische Einflüsse, können sich unterschiedliche Gefälle der SrBodenzusammensetzung bilden, wodurch Verbindung zwischen dem Boden 87Sr/86Sr-Verhältnis und dem Isotopenverhältnis der darauf wachsenden Pflanze, nicht mehr so eindeutig sein kann [15].

Diese Studie setzte sich zum Ziel, Auswirkungen externer (besonders landwirtschaftlich eingebrachter) Strontium-Quellen auf das 87Sr/86Sr-Verhältnis des Bodens und der darauf wachsenden Pflanzen nachzuweisen, die Verteilung des Sr-Isotopenverhältnisses in der Pflanze und den einzelnen Pflanzenteilen und den Einfluss des Bodens auf das Sr-Verhältnis zu erforschen und die Möglichkeiten der Analyse zur Unterscheidung von Äpfeln aus unterschiedlichen Anbaugebieten in Norditalien zu testen. Dafür wurden einjährige Apfelbäume (Malus $x$ domestica) in Töpfen unter kontrollierten Bedingungen, über zwei Wachstumsperioden unterschiedlichsten in der Landwirtschaft üblichen Behandlungen unterzogen. Dadurch konnte der Effekt einer Übertragung des Strontiums untersucht werden. In einem weiteren Experiment wurde in zwei verschiedenen Obstanlagen im Vinschgau und Unterland die Auswirkung des $87 \mathrm{Sr} / 86 \mathrm{Sr}$-Verhältnisses im Boden auf das Isotopenverhältnis in
Apfelbäumen untersucht. Zusätzlich wurden für jeden Standort die Unterschiede im Isotopenverhältnis von Proben untersucht und verglichen, die alle vom selben Teil desselben Apfelbaumes stammen (,intrapart variability"), von unterschiedlichen Teilen desselben Apfelbaumes (,intratree variability“) und auch noch jene die von unterschiedlichen Bäumen stammen („,intertree variability"). Im Anschluss wurden die Analysen auf 41 Standorte in Italien ausgeweitet, um zu sehen ob sich das $87 \mathrm{Sr} / 86 \mathrm{Sr}$-Verhältnis eignet, die Herkunft von Äpfeln aus verschiedenen italienischen Anbaugebieten (mit und ohne geschützter Ursprungsbezeichnung) zu unterscheiden.

\section{METHODEN}

\section{REAGENZIEN}

Ammoniumnitrat $\geq 98 \%$, Ammoniumsulfat $\geq 99 \%$, Bariumnitrat $\geq 99 \%$, Borsäure $\geq 99,5 \%$, Kupfersulfat pentahydrat $\geq 98 \%$, Ethylenediaminetetraessigsäure $\geq 98 \%$, Magnesiumsulfat wasserfrei $\geq 98 \%$, Manganchlorid tetrahydrat $\geq 98 \%$, Molybdänsäure $\geq 85 \%$, Kaliumnitrat Zellkulturqualität, Kaliumphosphat einbasig $\geq 99 \%$, Strontiumnitrat $\geq 99 \%$ und Zinksulfat heptahydrat $\geq 99 \%$ wurden bei Sigma-Aldrich erworben. Eisensulfat heptahydrat $\geq 98 \%$ wurde erworben bei J.T. Baker und Salpetersäure 65\% analytische Qualität bei Merck. Celluloseacetat Filter $(0,45 \mu \mathrm{m})$ und Polypropylen (PP)-Einwegfläschchen wurden erworben bei Vetrotecnica Srl, Polytetrafluoroethylen (PTFE) Filter $(0,45 \mu \mathrm{m})$ bei Thermo Fisher Scientific. Die Trennung des Strontiums von anderen Elementen wurde mit Strontium-spezifischen Resin (Srspec), erworben bei TrisKem International, durchgeführt. Für die Herstellung der Kalibrationskurve am induktiv-gekoppelten Plasma Massenspektrometer (ICP-MS) wurden monoelementare, zertifizierte analytische Standards aus Rubidium $(\mathrm{Rb})$ und Strontium (Sr) (erworben bei ULTRA Scientific) und Calcium (erworben bei Agilent Technologies) verwendet. Als interne Standards wurden Lösungen mit folgenden monoelementaren analytischen Standards vorbereitet: Scandium (Sc), Germanium (Ge) und Yttrium $(\mathrm{Y})$, alle erworben bei Inorganic Ventures, Merck. Zum Überprüfen der Qualität beider Messungen (Quantifizierung und Bestimmung des Isotopenverhältnisses) wurde zertifiziertes Referenzmaterial verwendet: TMDA-54,5 (LabService Analytica Srl) für das ICP-MS und SRM 987 (NIST) für das Multikollektor (mc)-
ICP-MS Instrument. Salpetersäure (65\%, Merck) und hochreines deionisiertes Wasser $(18,2 \mathrm{M} \Omega \cdot \mathrm{cm}$, Elix-Millipore), zusätzlich gereinigt durch ein auskochendes duoPur Destillationssystem (Milestone), wurden während aller Experimente und analytischer Arbeiten verwendet. Alle verwendeten Behältnisse und Gefäße wurden vor Gebrauch in saurer Lösung gereinigt und gespült. Alle Reagenzien und Chemikalien wurden nach den Herstellerangaben gelagert.

\section{EXPERIMENT 1 - AUSWIRKUNG VON LANDWIRTSCHAFTLICHEN EINFLÜSSEN}

Im April 2016 wurden 53 einjährige Apfelbäume (Malus X domestica Borkh, cv. Golden Delicious Klon B Lb, Unterlage M9, gekauft in einer Südtiroler Baumschule) im Gewächshaus der Freien Universität Bozen am Versuchszentrum Laimburg (Pfatten, BZ) eingetopft [28]. Jeder Baum wurde in einen Topf mit $18 \mathrm{~kg}$ luftgetrockneter Erde mit einer sandig-lehmigen Textur (61\% Sand, 38\% Schluff, 1\% Lehm, entnommen aus dem Flussufer der Etsch und gesiebt durch ein $2 \mathrm{~mm}$ Sieb) gepflanzt. Die Strontiumkonzentration des Bodens betrug 5,46 \pm $0,53 \mu \mathrm{g} / \mathrm{g}$ und das $87 \mathrm{Sr} / 86 \mathrm{Sr}$-Isotopenverhältnis 0,71256 $\pm 0,00007$. Die Bäume wurden über zwei Wachstumsperioden, von April 2016 bis Oktober 2017 in denselben Töpfen belassen. Im Winter 2016 wurde der Winterschnitt durchgeführt. Um eine $\mathrm{Sr}$ Kontamination durch Wettereinflüsse zu vermeiden blieben die Bäume im Gewächshaus. Sie wurden nach dem Zufallsprinzip in vier Gruppen unterteilt, jede Gruppe wurde einer anderen landwirtschaftlichen Behandlung unterzogen. Für die Kontrollgruppe (CTRL) war die Erde die einzige Strontiumquelle, die Bäume wurden mit destilliertem Wasser bewässert, mit einer im Labor hergestellten Sr-freien Nährstofflösung gedüngt und die Blätter mit destilliertem Wasser besprüht. Bei den anderen drei Gruppen wurde jeweils eine externe, praxisnahe Strontiumquelle hinzugefügt: entweder Leitungswasser (TW), ein kommerziell erhältlicher N-P-K Bodendünger (Triabon $16+8+$ 12) (SF) oder ein im Handel erhältlicher Calciumchlorid $(\mathrm{CaCl} 2)$ Blattdünger (Caso $\mathrm{FCC}$ Flakes) verdünnt mit destilliertem Wasser (FF). Zusätzliche Informationen über die Versuchsanordnung (Tab. 1). 
Tab. 1: Details zu den an den Apfelbäumen angewandten Behandlungen im Experiment 1 [28] // Details of treatments applied to apple trees in experiment 1 [28].

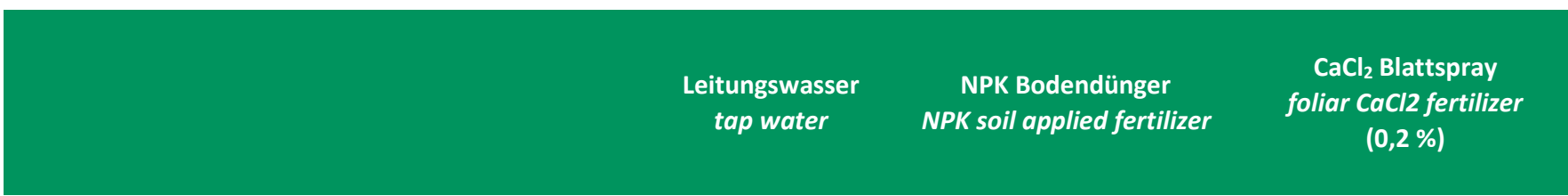

Anzahl Anwendungen 2016

no. applications in 2016

100

115

no. applications in 2017

Hinzugefügte Wassermenge (pro Baum)

Supplied water amount (total per tree)

Gesamtmenge an $\mathrm{Sr}$ (mg/Baum)

total amount of $\mathrm{Sr}(\mathrm{mg} / \mathrm{tree})$

${ }^{87} \mathrm{Sr} /{ }^{86} \mathrm{Sr}$-Verhälnis des zugegebenen Mediums

${ }^{87} \mathrm{Sr} /{ }^{86} \mathrm{Sr}$-Ratio of the added medium
$80 \mathrm{~g}$

$4.8 \mathrm{~L}$

2,9

0,8
Alle Bäume der Gruppen CTRL, TW und FF wurden mit der selbst hergestellten Strontium-freien Nährstofflösung gedüngt. Gleichzeitig wurden Bäume der SF-Gruppe mit dem Bodendünger gedüngt. Die $\mathrm{CaCl} 2-$ Lösung $(0,2 \%)$ wurde direkt auf die Baumkrone gespritzt, während dieses Vorgangs wurden die Bäume der FF-Gruppe von den anderen Gruppen durch einen Plastikvorhang getrennt, um eine Kontamination zu vermeiden. Zu Beginn des Experiments wurden die Erde, der kommerzielle Dünger und das $\mathrm{CaCl}$-Blattspray auf ihren Strontiumgehalt und $87 \mathrm{Sr} / 86 \mathrm{Sr}$ Isotopenverhältnis analysiert. Während des Experimentes wurde immer dieselbe Menge an Bodendünger und Blattspray verwendet. Das Leitungswasser wurde monatlich analysiert, aber es konnten keine signifikanten Änderungen in der Strontium Konzentration und im Isotopenverhältnis festgestellt werden. Zusätzlich wurden die Stämme und Äste von fünf Bäumen zu Beginn analysiert, um ihr anfängliches $87 \mathrm{Sr} / 86 \mathrm{Sr}$ Isotopenverhältnis festzustellen und um zu erkennen, ob es zu signifikanten Änderungen des Verhältnisses während der unterschiedlichen Behandlungen (Kontrolle, Leitungswasser, Bodendünger, Blattspray) kommt. Am Ende jeder Wachstumsperiode wurden sechs Bäume aus jeder Gruppe ausgewählt, und ihre Blätter, Zweige und Stämme wurden getrennt auf ihre $\mathrm{Sr}$ Konzentration und Isotopenverhältnis analysiert. Ein Teil des Bodens (ca. 2 kg) wurde ebenfalls analysiert. Im Verlauf des Experiments wurden während der beiden Wachstumsperioden regelmäßig Blattproben (je 10 apikale Blätter pro Baum von verschiedenen Ästen, zufällig ausgewählt) gesammelt und analysiert, um Veränderungen im 87Sr/86Sr-Isotopenverhältnis im Jahresverlauf zu ermitteln.

\section{EXPERIMENT 2 - AUSWIRKUNGEN} DES STANDORTS, UNTERSCHIEDE ZWISCHEN DEN BAUMBESTANDTEILEN INNERHALB EINES BAUMES UND ZWISCHEN DEN BÄUMEN

Dieses Experiment [29] wurde in zwei unterschiedlichen Obstwiesen an zwei Standorten in Südtirol durchgeführt (Vinschgau, Unterland). In jedem Feld befanden sich ausschließlich mehrjährige Apfelbäume (Malus X domestica Borkh., Gala, Klon Gala Schnitzer Schniga, Unterlage M9). Der Abstand zwischen den Bäumen in einer Reihe betrug $0,8 \mathrm{~m}$ und der Abstand zwischen den Reihen $3 \mathrm{~m}$. Die Apfelanlagen wurden nach den Empfehlungen der Südtiroler Richtlinien für integrierten Obstbau AGRIOS [30] kultiviert. Der Boden unter den Bäumen (ein schmaler Streifen von ca. $60 \mathrm{~cm}$ ) wurde mit Herbiziden behandelt, um das Wachstum von Gräsern und Unkräutern zu regulieren. Auf den Wegen zwischen den Reihen wurde das Gras gemulcht. Die erste Anlage (L) wurde 2010 gepflanzt und befindet sich im Unterland (Etschtal), südlich von Bozen, in der Nähe der Laimburg [geographische Koordinaten, $46^{\circ} 22^{\prime} 48.0^{\prime \prime} \mathrm{N}$ und $11^{\circ} 17^{\prime} 26.2^{\prime \prime}$ E; Höhenlage, $226 \mathrm{~m}$ ü. NN; Fläche des Blocks, ca. $16500 \mathrm{~m}^{2}$ ]. Für die Tropfberegnung wurde Grundwasser verwendet. Der Boden hat eine schluffig-lehmige Textur (25\% Sand, 64\% Schluff, 11\% Lehm), 2,7\% organischen Kohlenstoff, einen hohen Karbonatanteil und einen $\mathrm{pH}$-Wert in $\mathrm{CaCl} 2$ von 7,7. Die zweite Anlage (S), wurde 2007 angelegt und befindet sich im Vinschgau in der Nähe von Schluderns (geographische Koordinaten, $46^{\circ} 39^{\prime} 24.7^{\prime \prime} \mathrm{N}$ und $10^{\circ} 34^{\prime} 37.1^{\prime \prime} \mathrm{E}$ Höhenlage $900 \mathrm{~m}$ ü. NN; Fläche des Blocks, ca. $700 \mathrm{~m}^{2}$ ). Grundwasser wurde hier für die Überkronen-Sprinklerberegnung verwendet. Der Boden hat eine lehmige Textur (44\% Sand, 44\% Schluff und 12\% Lehm), $4,3 \%$ organischer Kohlenstoff, niedriger Karbonatanteil und einen $\mathrm{pH}$-Wert in $\mathrm{CaCl} 2$ von 5,9. Geologisch liegen beide Standorte in einem Bereich mit jungen Alluvialböden [31] Zusätzlich wurde dokumentiert, dass 


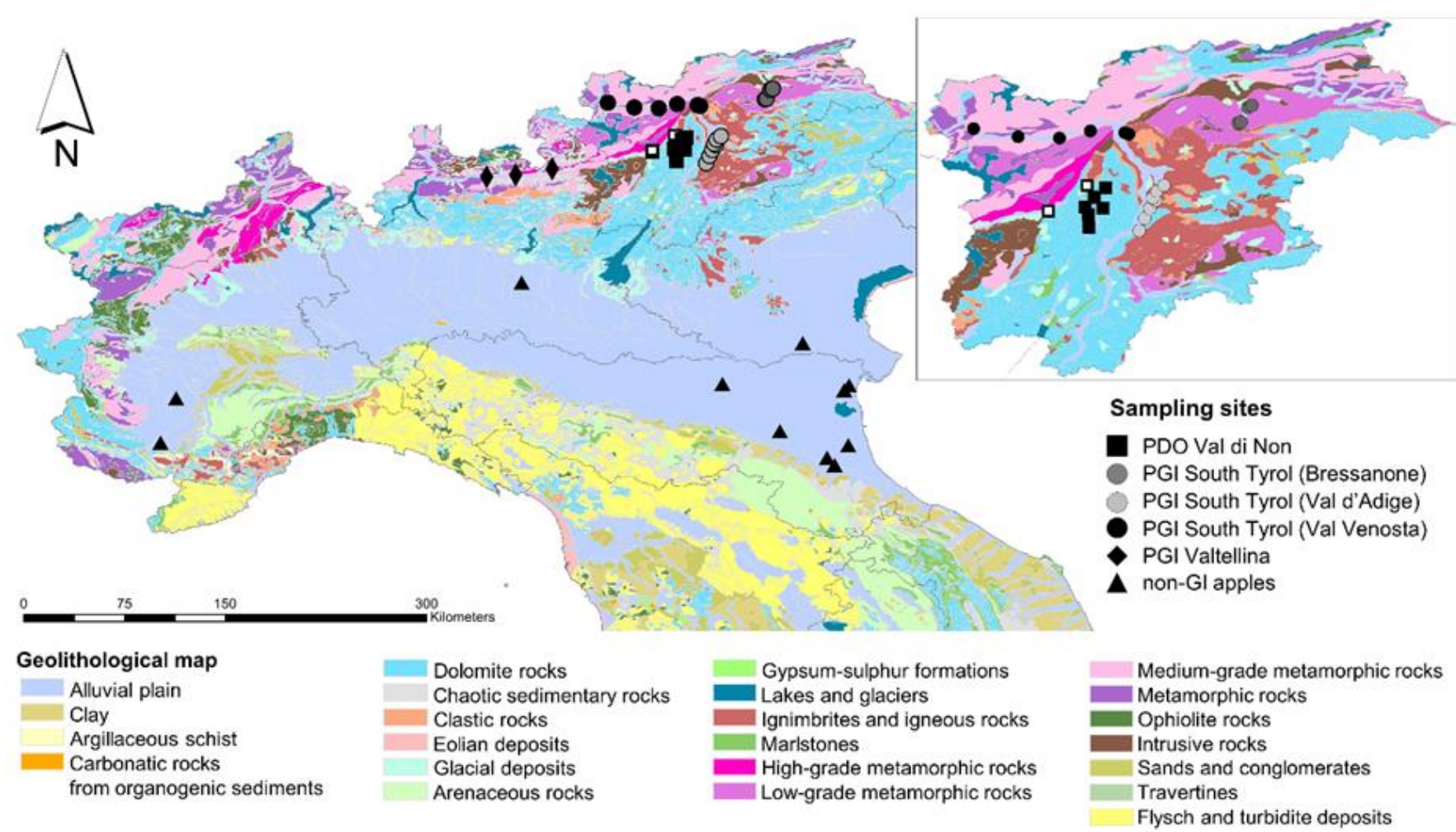

Abb. 2: Probennahmepunkte von Apfel- und Bodenproben dargestellt auf einer geologischen Landkarte (Quelle: http://www.pcn.minambiente.it) zwei Standorte in Val di Non (weißes Kästchen) wurden von der statistischen Varianzanalyse ausgeschlossen. // Sampling points of apple and soil samples on a geological map (source: http://www.pcn.minambiente.it), open squares represent the two orchards in Val di Non excluded from the analysis of variance. Source: [33], p. 3668.

zwischen 1930 und 1940 die Anlage S mit Erde aus einem Seitental aufgefüllt wurde.

Zum Erntezeitpunkt (August-September 2016) wurden jeweils sechs Apfelbäume, welche gleichmäßig in den Obstanlagen verteilt waren, ausgewählt. Zwei der sechs Bäume befanden sich jeweils nebeneinander in der gleichen Reihe. Von jedem Baum wurden sechs Triebe und sechs Früchte mit einem Abstand zum Boden von 1-2 m, von verschiedenen Ästen geerntet. An jedem Baum wurde an drei Punkten eine Bodenprobe entnommen, in einem Abstand zum Baumstamm von ca. $30 \mathrm{~cm}$. Wenn der Boden von den zwei benachbarten Bäumen entnommen wurde, wurde die Probe aus einer mit Gras bewachsenen Fläche zwischen den beiden Bäumen entnommen, mit einem Abstand von $20-40 \mathrm{~cm}$ vom Baumstamm. Der Bodenkern wurde in einer Tiefe von 10$20 \mathrm{~cm}$ mit Hilfe eines Bodenkernentnehmers (5 cm Durchmesser) entnommen [32]. Alle Bodenproben wurden getrennt aufgearbeitet und analysiert. Im Mai 2017 wurde eine zweite Bodenprobennahme durchgeführt. Dabei wurden in einer Tiefe von $80 \mathrm{~cm}$ zwischen drei pro Anlage zufällig ausgewählten Paaren von benachbarten Bäumen, in einem gleichmäßigen Abstand, Bodenproben entnommen. Die ersten $10 \mathrm{~cm}$ wurden verworfen, die verbleibenden wurden in Kompartimente im Abstand von $10 \mathrm{~cm}$ eingeteilt und dem entsprechend 7 Bodenproben im $10 \mathrm{~cm}$ Abstand bis $80 \mathrm{~cm}$ entnommen. Von jedem Baumpaar wurden je 10 Blätter pro Baum entnommen (zufällig ausgewählt) und dann zusammengelegt, um eine gesammelte Probe zu erhalten, welche mit dem Boden Sr-Profil verglichen werden konnte.

\section{EXPERIMENT 3 - ERMITTLUNG DES SR-ISOTOPENVERHÄLTNISSES VON} 41 STANDORTEN IN NORDITALIEN

Apfel- und Bodenproben wurden in zwei aufeinanderfolgenden Jahren (zwischen August und September 2017 und 2018) aus 41 unterschiedlichen Anlagen in Norditalien entnommen (Abb. 2) [33]. Alle gewählten Standorte sind in Tabelle 2 aufgelistet. In allen Obstanlagen wurde dieselbe Apfelsorte kultiviert (cv. Golden Delicious, Unterlage M9, mehrjährig). Die Probennahme erfolgte zwei Wochen vor der regulären Ernte (August-September). Die in der Studie ausgewählten Obstanlagen sind auf fünf Regionen in Norditalien verteilt: Trentino-Südtirol, Emilia Romagna, Lombardei, Piemont und Veneto. Die Anzahl der ausgewählten Obstanlagen variiert in jeder Region je nach Ausbreitung des Anbaubereichs. Äpfel aus dem Vinschgau (inklusive zwei Anlagen in der Nähe von Meran), Brixen und Umgebung und südlich von Bozen (Etschtal) trugen die Kennzeichnung „geschützte geographische Angabe“" jene aus Val di Non und Val di Sole (Trentino) waren mit "geschützte Ursprungsbezeichnung" gekennzeichnet, ebenso wie die Äpfel aus der Valtellina (Lombardei). Äpfel ohne Kennzeichnung stammten aus 13 Anlagen in den Regionen Emilia Romagna, Lombardei, Piemont und Veneto.

2017 wurden Apfelproben aus 41 Anlagen entnommen. Aus jeder Anlage wurden von 10 gleichmäßig in der Wiese verteilten Bäumen jeweils drei Äpfel geerntet (drei Äpfel pro Baum). Sie wurden aus einer Höhe von 1-2 m über dem Boden entnommen. 2018 wurde die Probennahme in 20 der bereits untersuchten Anlagen wiederholt, dieses Mal wurden Apfel- und Bodenproben entnommen. Die Anlagen aus 2018 wurden so ausgewählt, dass sie den gesamten 
Tab. 2: Auflistung aller 41 ausgewählten Standorte für die Probennahme in Norditalien // List of all 41 selected locations for sampling in Northern Italy.

\begin{tabular}{|c|c|c|c|}
\hline $\begin{array}{l}\text { Anbaugebiet } \\
\text { Growing area }\end{array}$ & $\begin{array}{l}\text { Standort } \\
\text { Location }\end{array}$ & $\begin{array}{l}\text { Anbaugebiet } \\
\text { Growing area }\end{array}$ & $\begin{array}{l}\text { Standort } \\
\text { Location }\end{array}$ \\
\hline \multirow{6}{*}{$\begin{array}{l}\text { Vinschgau und } \\
\text { Burggrafenamt }\end{array}$} & Schluderns & \multirow[t]{2}{*}{ Piemonte } & Cuneo \\
\hline & Kortsch & & Savigliano \\
\hline & Kastelbell & \multirow[t]{7}{*}{ Emilia Romagna } & San Agostino \\
\hline & Plaus & & Sesto Imolese \\
\hline & Fragsburg & & Comacchio 1 \\
\hline & Sinich & & Comacchio 2 \\
\hline \multirow[t]{4}{*}{ Natz-Schabs } & Albeins & & San Bartolo \\
\hline & Sarns & & Roncadello \\
\hline & Elvas & & Bagnolo \\
\hline & Natz & \multirow[t]{8}{*}{ Val di Non } & Rumo \\
\hline \multirow[t]{7}{*}{ Unterland } & Leifers & & Brez \\
\hline & Pfatten & & Revo \\
\hline & Laimburg & & Mechel \\
\hline & Auer & & Coredo \\
\hline & Binnenland & & Tuenno \\
\hline & Auer & & Termon \\
\hline & Salurn & & Presson \\
\hline \multirow[t]{4}{*}{ Lombardia } & Berbenno Valt & \multirow[t]{4}{*}{ Veneto } & Eraclea \\
\hline & Ponte Valt & & Ca Tron \\
\hline & Lovero & & Ceregnano \\
\hline & Corzano & & \\
\hline
\end{tabular}

Probennahmebereich und das gesamte 87Sr/86Sr-Isotopenverhältnis der Äpfel, geerntet im Jahr 2017, abdecken. Aus jeder Anlage wurden fünf Bäume ausgewählt und die Probennahme erfolgte auf dieselbe Weise wie 2017. Bodenkernproben (in einer Tiefe von $10-40 \mathrm{~cm}$ unter der Oberfläche) wurden in einem Abstand von jeweils $30 \mathrm{~cm}$ von den beprobten Apfelbäumen, mit Hilfe einer Bodenschnecke (einteilig, Edelman Typ) entnommen.

\section{PROBENVORBEREITUNG}

Die Bodenproben wurden durch ein $2 \mathrm{~mm}$ Sieb gesiebt und alle Wurzelrückstände manuell entfernt. Im Anschluss wurden die Proben bei $40^{\circ} \mathrm{C}$ für $48 \mathrm{~h}$ getrocknet. Das bioverfügbare Strontium wurde mit $1 \mathrm{M}$ NH4NO3 extrahiert (10 g Bodenprobe mit
25 ml NH4NO3, 2 h schütteln bei Raumtemperatur, zentrifugiert bei $2400 \mathrm{rpm}$ für 5 Min.), nach DIN ISO 19730 [34].

Baumstämme, Triebe, Äste und Blätter der Apfelbäume (alle getrennt behandelt, Blätter wurden von den Ästen getrennt) wurden mit destilliertem Wasser gewaschen, um Staub und Partikel zu entfernen (mit Ausnahme jener Proben, welche mit N-P-K Dünger behandelt wurden), im Ofen bei $65^{\circ} \mathrm{C}$ für $48 \mathrm{~h}$ getrocknet und im Anschluss pulverisiert (Schwingmühle Retsch MM400). Die Äpfel wurden ebenfalls mit destilliertem Wasser abgewaschen, geschält, das Kerngehäuse entfernt und in $1 \mathrm{~cm}$ dicke äquatoriale Scheiben geschnitten. Das Apfelfruchtfleisch und die Schale wurden gefriergetrocknet (FreeZone, Labconco) und pulverisiert. $0,5 \mathrm{~g}$ von jeder pulverisierten Probe
(Frucht, Triebe, Äste, Stämme und Blätter) wurden mit jeweils $5 \mathrm{ml} \mathrm{HNO3}(65 \%$, w/w) in einem Milestone UltraWAVE Gerät mineralisiert (Details siehe Aguzzoni et al. [28]). Die verdauten Proben wurden durch einen PTFE-Filter gefiltert $(0,45 \mu \mathrm{m})$ und mit destilliertem Wasser auf ein Volumen von $10 \mathrm{ml}$ verdünnt.

Um die Elemente zu entfernen, welche mit dem Strontium während der Analyse interferieren können (insbesondere $\mathrm{Ca}$ und $\mathrm{Rb}$ ), wurde das Sr mit Hilfe von Sr-spezifischem Resin (Triskem International) manuell abgetrennt. Die Methode nach Swoboda et al. [35], mit kleineren Abänderungen, wurde für dieses Verfahren angewandt. Zuerst wurde das Resin mit 5,5 ml $8 \mathrm{M} \mathrm{HNO3} \mathrm{akti-}$ viert, die mit $\mathrm{HNO} 3(8 \mathrm{M})$ verdünnte Probe wurde aufgetragen und im Anschluss mit 
HNO3 (8 M) gewaschen. Zum Schluss wurde das $\mathrm{Sr}$ aus der Kolonne mit $8 \mathrm{ml}$ destilliertem Wasser wiedergewonnen. Anschließend wurde die effektive Sr/Matrix-Trennung mittels ICP-MS auf den Gehalt an Sr überprüft. Durch anschließende Verdünnung wurde die Konzentration der Sr-Lösungen auf 200 ng/g eingestellt, mit Ausnahme der Lösungen von Apfelfruchtfleisch und Schale welche auf $20 \mathrm{ng} / \mathrm{g}$ eingestellt wurden.

\section{ICP-MS UND MC-ICP-MC ANALYSE}

Die Quantifizierung des Strontiums erfolgte mittels eines induktiv-gekoppelten Plasma Massenspektrometers (iCAP Q ICP-MS, Thermo Scientific, Bremen, Deutschland) ausgestattet mit einem Autosampler ASX520 (Cetac Technologies, Inc., Omaha, NE, USA). Stammlösungen der Standards wurden verdünnt, um eine Kalibriergerade mit folgenden Konzentrationen zu erstellen: $0,01-250 \mathrm{ng} / \mathrm{g}$ für $\mathrm{Rb}$ und $\mathrm{Sr}$ und 0,01-50 $\mu \mathrm{g} / \mathrm{g}$ für $\mathrm{Ca}$. Folgende Isotope wurden quantifiziert: $44 \mathrm{Ca}, 85 \mathrm{Rb}$ und $88 \mathrm{Sr}$. Als interner Standard wurde eine Lösung aus $Y$, Sc und Ge hinzugefügt. Die Genauigkeit des Instruments wurde durch das Messen eines zertifizierten Referenzmaterials TMDA-64.3 bestimmt und betrug im Durchschnitt zwischen 90 und $110 \%$.

Das $87 \mathrm{Sr} / 86 \mathrm{Sr}$ wurde mittels eines doppelfokussierenden Multikollektoren induktivgekoppelten Plasma-Massenspektrometers (mc-ICP-MS) (Neptune Plus, Thermo Scientific Bremen, Deutschland) mit einer NierJohnson Geometrie, analysiert. Die Analyse wurde im statischen Modus und mit niedriger Auflösung durchgeführt. Der Multikollektor bestand aus insgesamt neun Farraday-Cups, acht davon waren beweglich und eine im Zentrum fixiert. Das Instrument wurde täglich justiert (um die Sensitivität zu erhöhen und die Peakform zu verbessern) und die Genauigkeit wurde durch das Messen des zertifizierten NIST SRM 987 Referenzmaterials zu Beginn und am Ende jeder Probensequenz [36] [37], inklusive mehrere Blindwerte, überprüft. Die Rohdaten wurden folgendermaßen korrigiert: der Blindwert wurde subtrahiert, die Massenausrichtung wurde korrigiert durch Normalisieren des $87 \mathrm{Sr} / 86 \mathrm{Sr}$-Wertes auf den von der International Union of Pure and Applied Chemistry (IUPAC) vorgegeben Wert von 8,3752 [38] und die isobarische Interferenz von $86 \mathrm{Kr}$ und $87 \mathrm{Rb}$ wurde mathematisch korrigiert [32]. Boden-, Triebe und Blätterproben wurden mittels eines trockenen Aerosols (CETAC Aridus apparatus als Aerosol
Trockenvorrichtung und jet sample cone $+\mathrm{H}$ Ni Skimmer cone) ins Plasma eingebracht, Fruchtfleisch- und Schalenproben mittels eines feuchten Aerosols. Alle analysierten Lösungen wurden mit Salpetersäure $(65 \%$ w/w) auf eine Konzentration von 2\% HNO3 eingestellt. Die Methode wurde von Aguzzoni et al. [28] validiert.

\section{STATISTISCHE AUSWERTUNG}

Alle statistischen Auswertungen und Analysen wurden mit der Software R (R Development Core Team, 2016) durchgeführt.

\section{EXPERIMENT 1}

Um statistisch signifikante Unterschiede zwischen den vier unterschiedlichen Behandlungen zu ermitteln, wurde eine Varianzanalyse (ANOVA) durchgeführt. Der posthoc Tukey HSD (Honest Significant Difference) Test wurde für paarweise Vergleiche angewandt. Nicht-parametrische Tests (Kruskal-Wallis mit "multiple bilateral comparison") wurden angewandt, wenn die inkludierten Gruppen nicht dieselbe Anzahl an Proben hatten oder eine andere Bedingung für einen parametrischen Test (Normalverteilung, homogene Varianzen) nicht erfüllt wurde [39].

\section{EXPERIMENT 2}

Unterschiede im 87Sr/86Sr-Isotopenverhältnis zwischen den verschiedenen Proben desselben Baumbestandteiles („intrapart variability") wurde anhand der Streuung des Isotopenverhältnisses um den Mittelwert für jeden Baumbestandteil (Trieb, Blatt, Frucht) bewertet. Eine Varianzanalyse (ANOVA) wurde angewandt, um die 87Sr/86Sr-Isotopenverhältnisse zwischen den einzelnen Bestandteilen eines Baumes miteinander zu vergleichen (,intratree variability") und um anschließend das Isotopenverhältnis zwischen den verschiedenen Bäumen zu vergleichen (,intertree variability“). Der posthoc Tukey HSD Test wurde für paarweise Vergleiche angewandt. Ein Zweistichproben $\mathrm{t}$-Test für abhängige Stichproben wurde verwendet, um das Isotopenverhältnis der Blätter mit dem des Bodens zu vergleichen $(p=0,05)$.

\section{EXPERIMENT 3}

Der Kruskal-Wallis und der Dunn post hoc Test (mit Bonferroni Korrekturen) wurden verwendet, um Mittelwerte der Isotopenanalyse (der verschiedenen Anbaugebiete) zu vergleichen. Eine nicht-parametrische Varianzanalyse wurde ebenfalls angewandt, da die beiden Vergleichsgruppen nicht dieselbe Anzahl an Proben hatten, sowie mindestens eine Voraussetzung für einen parametrischen Test nicht erfüllt wurde. Für diesen Vergleich wurden die drei Hauptanbaugebiete in Südtirol (Vinschgau, Brixen und Umland, Etschtal) als getrennte Gruppen betrachtet. Um Unterschiede im Isotopenverhältnis zwischen den beiden Jahrgängen 2017 und 2018 zu bestimmen, wurden eine lineare Korrelationsanalyse und ein zweiseitiger t-Test für abhängige Stichproben durchgeführt. Die lineare Korrelationsanalyse wurde auch für die Isotopenverhältnisse der Äpfel und des Bodens angewandt. Der $p$-Wert wurde auf 0,05 festgesetzt.

\section{ERGEBNISSE UND DISKUSSION}

\section{EXPERIMENT 1 - AUSWIRKUNGEN LANDWIRTSCHAFTLICHER EIN- FLÜSSE AUF DAS SR-ISOTOPEN- VERHÄLTNIS}

Die Strontiumkonzentration im Baumstamm und in den Ästen betrug zum Zeitpunkt des Einpflanzens der Apfelbäume 15,0 \pm $3,3 \mu \mathrm{g} / \mathrm{g}$ (Äste) und 7,4 $\pm 1,5 \mu \mathrm{g} / \mathrm{g}$ (Stamm) [28]. Trotz dieser Unterschiede in der SrKonzentration, wiesen beide ein ähnliches 87Sr/86Sr-Isotopenverhältnis auf (Durchschnittswerte von $0,71030 \pm 0,00003$ für den Stamm und 0,71028 $\pm 0,00002$ für die Äste). Ähnliche Ergebnisse gehen auch aus der Literatur hervor [40] [41]. Diese Isotopenzusammensetzung unterschied sich signifikant $(p<0,001)$ von jener der anderen SrQuellen aus dem Experiment (Boden, Leitungswasser, Bodendünger, Blattspray). Die hinzugefügten Gesamtmengen an $\mathrm{Sr}$ im Boden oder direkt auf die Pflanze sind in Tabelle 1 zusammengefasst. Zu Beginn befanden sich ca. $90 \mathrm{mg}$ Sr pro Topf in der Erde. Bei den Blatt- und Triebproben gab es keine signifikanten Unterschiede $(p>0,05)$ in der Sr-konzentration, zwischen den unterschiedlichen Behandlungen (jeweils am Ende der ersten und der zweiten Wachstumsperiode). In Tabelle 3 sind die Ergebnisse der Pflanzen- und Bodenisotopenzusammensetzung zusammengefasst. Bei der Probennahme im Oktober 2016 wurde das 87Sr/86Sr-Isotopenverhältnis im Boden bereits durch die Behandlungen beeinflusst. Insbesondere das Bewässern mit Leitungswasser führte zu einer signifikanten Erhöhung des $87 \mathrm{Sr} / 86 \mathrm{Sr}$-Verhältnisses im Boden im Vergleich zu den anderen Behandlungen 
Tab. 3: 87Sr/86Sr-Isotopenverhältnis der unterschiedlichen Organe des Apfelbaumes und des Bodens, entnommen jeweils am Ende jeder Wachstumsperiode und nach Behandlung sortiert, Experiment 1 [28]. CTRL = Kontrollgruppe, $\mathrm{TW}=$ Leitungswasser, $\mathrm{SF}=$ Bodendünger, $\mathrm{FF}=\mathrm{Blattspray} / /$ 87Sr/86Sr of different apple tree organs and soil collected at the end of each growing season and sorted per treatment, Experiment 1 [28]. CTRL $=$ control group, $T W=$ tap water, $S F=$ soil fertilizer, $F F=$ foliar fertilizer.

\begin{tabular}{|c|c|c|c|c|c|c|c|}
\hline $\begin{array}{l}\text { Jahr } \\
\text { year }\end{array}$ & $\begin{array}{l}\text { Gruppe } \\
\text { group }\end{array}$ & $\begin{array}{l}\text { Boden } \\
\text { soil }\end{array}$ & $\begin{array}{c}\text { Stamm } \\
\text { trunk }\end{array}$ & $\begin{array}{l}\text { Zweige } \\
\text { branches }\end{array}$ & $\begin{array}{l}\text { Triebe } \\
\text { shoots }\end{array}$ & $\begin{array}{l}\text { Blätter } \\
\text { leaves }\end{array}$ & $\mathbf{P}$ \\
\hline \multirow[t]{4}{*}{2016} & CTRL & $0,71294 b, x$ & $0,71183 a b, y$ & 0,71168 y & 0,71187 y & 0,71174 y & $* * *$ \\
\hline & TW & $0,71358 a, x$ & $0,71197 a, y$ & 0,71163 y & 0,71188 y & 0,71177 y & $* *$ \\
\hline & SF & $0,71301 b, x$ & $0,71177 \mathrm{ab}, \mathrm{y}$ & 0,71157 y & 0,71182 y & 0,71179 y & $* * *$ \\
\hline & $\mathrm{FF}$ & $0,71302 b, x$ & $0,71171 b, y$ & 0,71161 y & 0,71177 y & 0,71161 y & $* *$ \\
\hline$P$ & & $* *$ & $*$ & ns & ns & ns & \\
\hline \multirow[t]{4}{*}{2017} & CTRL & $0,71211 a b, x y$ & $0,71241 a b, x y$ & $0,71220 a b, y$ & $0,71254 a b, x y$ & $0,71253 a b, x$ & $*$ \\
\hline & TW & $0,71334 a, x$ & $0,71290 a, x y$ & $0,71265 a, y$ & $0,71287 a, x y$ & 0,71280 a,xy & $* *$ \\
\hline & SF & $0,71187 b, y$ & $0,71225 b, x y$ & $0,71212 b, y$ & $0,71244 b, x$ & $0,71244 b, x$ & $*$ \\
\hline & $\mathrm{FF}$ & $0,71181 b, z$ & $0,71240 a b, x y z$ & 0,71214 b,yz & $0.71246 b, x$ & $0,71245 b, x y$ & $*$ \\
\hline $\mathrm{P}$ & & $* *$ & $* *$ & $* *$ & $* *$ & $*$ & \\
\hline
\end{tabular}

Asterisks denote significance $*=0,05<P<0,01, * *=0.01<P<0,001, * * *=P<0,001$. ns $=$ not significant $(P>0,05)$

$\mathrm{a}, \mathrm{b}=$ different letters along columns denote significance among groups

$x, y, z=$ different letters along rows denote significance among tree organs and soil.

$(p<0,01)$. Dies stimmt auch mit der insgesamt zugefügten Menge (Konzentration) an Sr und der Isotopenzusammensetzung des insgesamt zur Bewässerung eingesetzten Leitungswassers überein (Tab. 1). Die Erde, die mit Leitungswasser bewässert wurde, zeigt als einzige eine signifikante Erhöhung $(p<0,05)$ des 87Sr/86Sr-Verhältnisses im Vergleich zum Verhältnis am Anfang des Experiments. Alle Pflanzenorgane, unabhängig von der Behandlung hatten 2016 noch ein Isotopenverhältnis, welches nicht mit dem aus dem Boden übereinstimmte, sich aber allerdings vom initialen Verhältnis in der Pflanze unterscheidet $(p<0,05)$.

Bei der finalen Probennahme im Oktober 2017 wies der mit Leitungswasser bewässerte Boden als einziger nur geringe Unterschiede im $87 \mathrm{Sr} / 86 \mathrm{Sr}$-Isotopenverhältnis zum Vorjahr auf $(p<0,05)$, insgesamt erhöhte sich das Verhältnis jedoch im Vergleich zum Vorjahr $(p<0,05)$. Der Boden der Kontrollgruppe, behandelt mit Bodendünger (SF) und jener behandelt mit Blattspray $(F F)$, zeigten keine signifikanten Veränderungen zum anfänglichen $87 \mathrm{Sr} / 86 \mathrm{Sr}$-Isotopenverhältnis $(p>0,05)$ (Abb. 3). Die leichte Verringerung des Isotopenverhältnisses im Boden im Jahr 2017 (im Vergleich zum Vorjahr) könnte durch unterschiedlichste Vorgänge im Boden ausgelöst worden sein. Ein Beispiel dafür wäre die Freisetzung von nicht radiogenem $\mathrm{Sr}$ durch die Karbonatfraktion des Bodens, wodurch sich das Isotopenverhältnis zugunsten der nicht radiogenen Isotope verschoben hat. Auch durch NährstoffRecycling oder der Zersetzung kleinerer Wurzeln der Pflanze kann dieses Phänomen verstärkt werden [42]. In den Pflanzenorganen erhöhte sich zwischen Oktober 2016 und 2017 das Isotopenverhältnis leicht $(p<0,01)$. Das finale Isotopenverhältnis der Pflanzenorgane der Kontrollgruppe zeigte jedoch keine signifikanten Unterschiede zum initialen Verhältnis des Bodens. Keine
Behandlung veränderte das $87 \mathrm{Sr} / 86 \mathrm{Sr}$-Isotopenverhältnis signifikant in den Pflanzenbestandteilen im Vergleich zur Kontrollgruppe $(p<0,05)$. Zu Beginn der neuen Wachstumsperiode im Frühling 2016 war das $87 \mathrm{Sr} / 86 \mathrm{Sr}$-Isotopenverhältnis in den neu gewachsenen Pflanzenorganen geringer als im Boden (Abb. 3). Ähnlich wie beim Transport von Ca im Baum, [43] [44], wird auch das $\mathrm{Sr}$ nur langsam von der Wurzel in das Blatt transportiert [41]. Aus diesem Grund könnte es Monate oder Jahre dauern bis

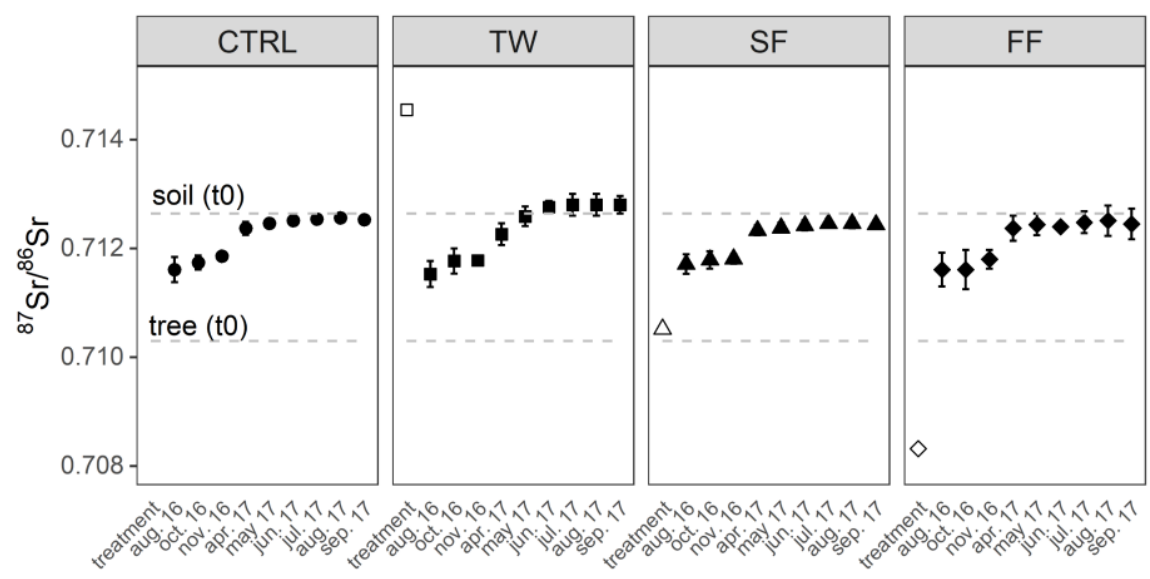

Abb. 3: Entwicklung des 87Sr/86Sr der Blattproben unterteilt nach Behandlungen, Mittelwert \pm Standardabweichung [28], CTRL = Kontrollgruppe, TW = Leitungswasser, $\mathrm{SF}=$ Bodendünger, $\mathrm{FF}=$ Blattspray // Evolution of the $875 r / 865 r$ of leaf samples sorted per group, Means value \pm standard deviations [28], CTRL = control group, TW = tap water, $S F=$ soil fertilizer, $F F=$ foliar fertilizer. 
Bäume die Isotopenzusammensetzung des Bodens, auf dem sie wachsen annehmen. Diese Verzögerung ist in unseren Daten erkennbar und könnte ein Grund sein wieso der Einfluss gewisser Behandlungen möglicherweise nicht erkannt wurde. Es ist daher ratsam, für zukünftige wissenschaftliche Experimente, den Bäumen ausreichend Zeit zu geben, um sich an das Substrat anzupassen und die Behandlungen erst zu starten sobald ein Gleichgewicht zwischen dem Isotopenverhältnis im Boden und der Pflanze erreicht ist.

\section{SCHLUSSFOLGERUNGEN}

Unsere Ergebnisse zeigen auf, dass die Pflanze nach einiger Zeit ein sehr ähnliches
Isotopenverhältnis wie das des Bodens annimmt. Dies ist eine der Hauptvoraussetzungen um das $87 \mathrm{Sr} / 86 \mathrm{Sr}$-Isotopenverhältnis als geographischen Indikator für landwirtschaftliche Produkte zu verwenden. Des Weiteren geht aus unseren Ergebnissen hervor, dass externe Sr-Quellen im Boden das Isotopenverhältnis der Pflanze nur schwach verändern. Aufgrund des langsamen $\mathrm{Sr}$ Transports in der Pflanze ist allerdings der Nachweis dieser Effekte nur begrenzt bzw. erst nach Ablauf einer bestimmten Zeit möglich. Bei den von uns untersuchten Apfelbäumen dauerte es zwei Wachstumsperioden bis die Pflanze das Isotopenverhältnis des Bodens annahm. Aus diesem Grund sind junge, erst vor kurzem eingepflanzte Bäume nicht für Sr-Isotopenverhältnis Herkunfts- studien geeignet. Trotz des Einflusses externer Sr-Quellen (Bodendünger, Blattspray, Beregnungswasser) kann das $87 \mathrm{Sr} / 86 \mathrm{Sr}$-Verhältnis aber als geographischer Indikator verwendet werden, um die Herkunft landwirtschaftlicher Produkte zu bestimmen. Allerdings muss dabei ein besonderes Augenmerk auf die Datenverarbeitung der Analysenergebnisse gelegt werden, um den $\mathrm{Zu}$ sammenhang mit den geologischen Eigenschaften des Herkunftsgebietes zu finden [41]. Mathematische Methoden können dabei behilflich sein, externe Einflüsse auf das Isotopenverhältnis zu beschränken [45] Auch muss bei Anwendung dieser mathematischen Methoden die genaue Herkunft des externen $\mathrm{Sr}$ bekannt sein, was bei unbekannten oder bearbeiteten Proben nur
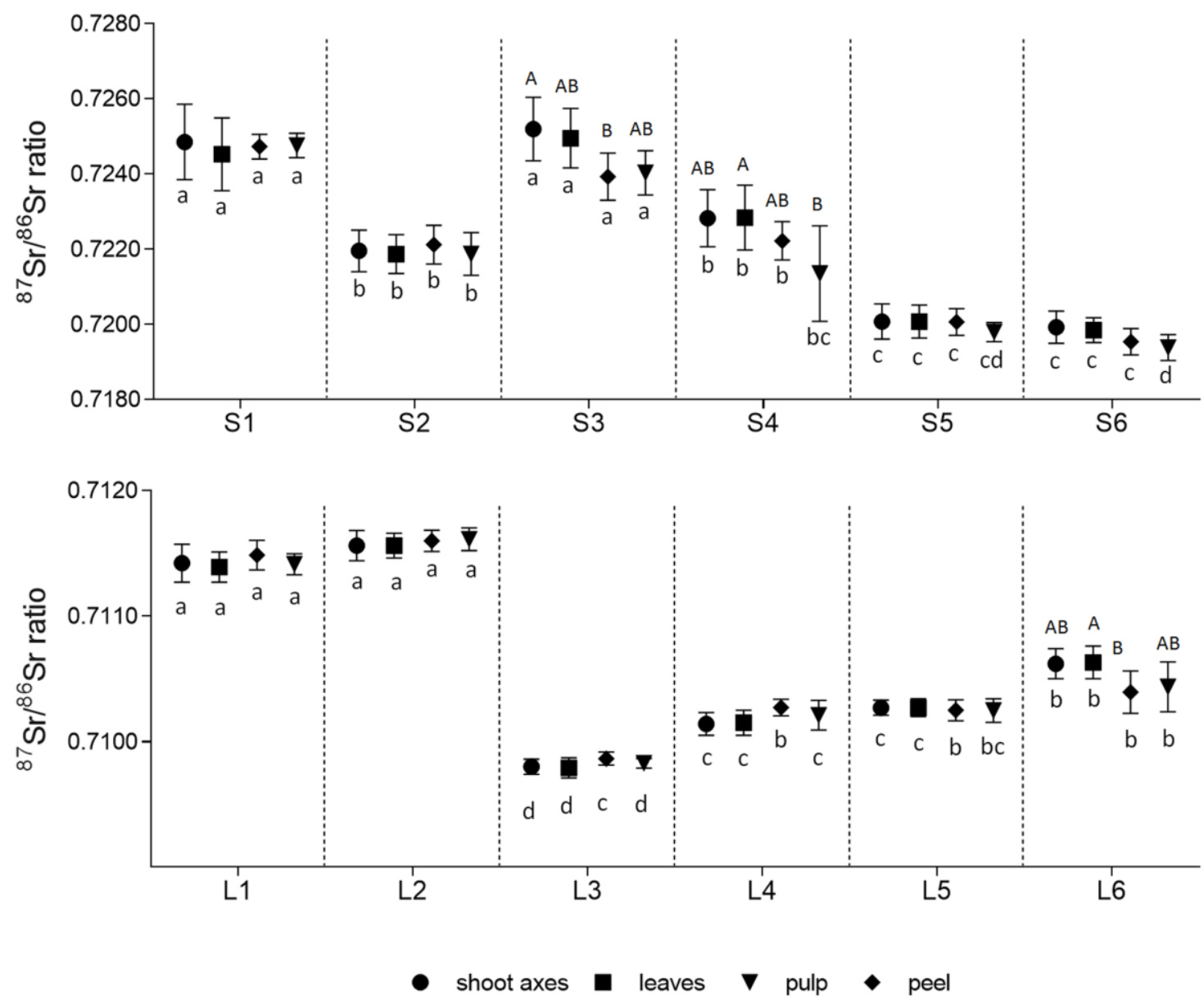

Abb. 4: 87Sr/86Sr Isotopenverhältnis gemessen in unterschiedlichen Pflanzenorganen (Triebe, Blätter, Apfelfruchtfleisch, Apfelschale) in sechs Apfelbäumen aus der Obstanlage Laimburg (L, obere Grafik, L1-L6) und Schluderns (S, untere Grafik, S1-S6). Die Punkte stehen für den Mittelwert \pm Standardabweichung der sechs Proben. Tiefgestellte Buchstaben zeigen signifikante Unterschiede zwischen demselben Organ/Gewebe zwischen den verschiedenen Bäumen auf, hochgestellte Buchstaben stehen für signifikante Unterschiede zwischen verschiedenen Organen/Gewebe innerhalb desselben Baumes. // 87Sr/86SR ratio measured in different organs (shoots axis, leaves, apple fruit flesh, apple fruit peel) of the six apple trees (L1-L6) sampled in the orchard Laimburg (L, upper graph, L1-L6) and Sluderno (S, downer graph, S1-S6). Each point represents the mean \pm standard deviation of six samples. Lowercase letters identify significant differences for the same tissue among different trees; upper case letters identify significant differences for different organs/tissues within the same tree.

Source: [29], p. 5730. 


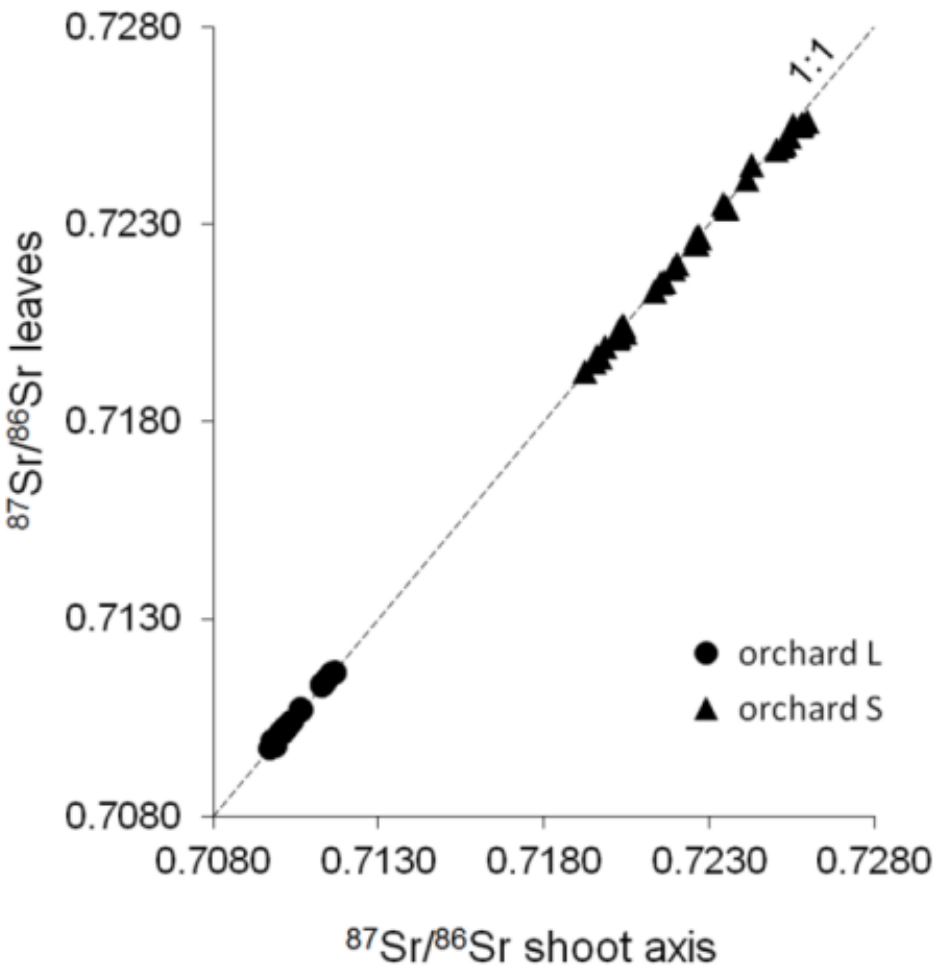

Abb. 5: 87Sr/86Sr-Isotopenverhältnis der Blätter aufgetragen gegen das 87Sr/86Sr-Verhältnis der Triebe an denen sie wuchsen. Jeder Punkt repräsentiert eine Probe $(n=36) / / 87 \mathrm{Sr} / 86 \mathrm{Sr} r a$ tio of the leaves plotted against the $87 \mathrm{Sr} / 86 \mathrm{Sr}$ ratio of the shoot axis where they were attached. Each point represents a sample $(n=36)$.

Source: [29], p. 5731.

teilweise bis gar nicht möglich ist [46]. Diese Aspekte sollten für zukünftige Anwendungen berücksichtigt werden.

\section{EXPERIMENT 2 - AUSWIRKUNGEN}

\section{DES STANDORTS, UNTERSCHIEDE}

\section{ZWISCHEN DEN BAUMBESTAND-}

\section{TEILEN INNERHALB EINES BAUMES} UND ZWISCHEN DEN BÄUMEN

In Abbildung 4 sind die 87Sr/86Sr-Isotopenverhältnisse der jeweils sechs Proben pro Pflanzenorgan, aus den beiden Obstanlagen von Laimburg (L) und Schluderns (S), aufgetrennt nach den einzelnen Bäumen, dargestellt [29]. In der Anlage $L$ reicht das 87Sr/86Sr-Isotopenverhältnis zwischen den verschiedenen Baumbestandteilen von 0,70970 bis $0,71173(\Delta=0,00203)$, in Anlage $S$ von 0,71895 bis $0,72598(\Delta=0,00703)$. Somit ist davon auszugehen, dass in der Anlage $S$ ein höherer Anteil von radiogenem $\mathrm{Sr}$ im Boden vorhanden ist. Das durchschnittliche Isotopenverhältnis, berechnet aus den Mittelwerten jedes untersuchten Baumes, betrug 0,71063 $\pm 0,00071$ für die Anlage $L$ und $0,72219 \pm 0,00214$ für Anlage $S$. Die
Verhältnisse aus Anlage $S$ wiesen eine größere Spannbreite auf als jene aus Anlage $L$ (Abb. 4). Dies lässt sich allerdings nicht mit der Größe der Anlage erklären, da der getestete Block mit Galaäpfeln in Anlage S, kleiner war als in Anlage L. Für beide Anlagen zeigte die ANOVA Analyse eine homogene Verteilung des Isotopenverhältnisses zwischen den verschiedenen Pflanzenteilen desselben Baumes (,intratree“) d.h. es gab keine signifikanten Unterschiede im 87Sr/86Sr zwischen den verschiedenen Pflanzenorganen (Triebe, Blätter, Apfelfruchtfleisch und Apfelschale) eines Baumes (Abb. 4). Diese Homogenität ist auch in Abbildung 5 gut erkennbar, in welcher die Isotopenverhältnisse der Blätter gegen jene der Triebe aufgetragen wurden. Hier zeigte sich eine sehr hohe Korrelation zwischen den beiden Organen (Triebe und dazugehörige Blätter) mit $\mathrm{R}>0$,99. Im Gegensatz dazu zeigte der Vergleich zwischen den gleichen Pflanzenorganen von verschiedenen Bäumen aus derselben Anlage (,intertree") eine geringere Homogenität $(p<0,001)$ für beide Obstanlagen (Abb. 4). Jedoch wiesen die jeweils benachbarten Bäume, in beiden Obstanlagen, dieselbe Isotopenzusammensetzung auf (Abb. 4, L1 und L2, S1 und S2). Um diese „intratree" Homogenität und „intertree" Heterogenität zu untersuchen wurde auch das Isotopenverhältnis des entsprechenden Bodens analysiert, dafür wurden jeweils drei Bodenproben (aus 10-20 cm Tiefe) direkt unter jedem beprobten Apfelbaum entnommen.

Bodenproben aus der Obstanlage $L$ hatten ein Isotopenverhältnis zwischen 0,70853 und 0,71098 , in Anlage S reichte das Verhältnis hingegen von 0,71424 bis 0,71804 (Tab. 4). Die Standardabweichung des 87Sr/86Sr-Isotopenverhältnisses der Bodenproben zeigte für Anlage $S$ eine größere $\mathrm{Va}$ riabilität als in Anlage L $(0,00094$ bzw. $0,00027)$. Diese Ergebnisse weisen auf eine größere Komplexität der Bodenmatrix in Obstanlage $\mathrm{S}$ hin und zeigen, dass das 87Sr/86Sr-Isotopenverhältnis im Boden, aus derselben Tiefe, selbst in einem kleinen Areal über einen breiten Bereich variieren kann. Dieser Effekt könnte auch durch das Einbringen von Bodenmaterial in den 30er Jahren aufgetreten sein.

Das durchschnittliche Isotopenverhältnis der Blätter pro Baum wurde mit den entsprechenden Bodenproben verglichen. Für den Datensatz aus Anlage L konnte eine hohe Korrelation $(R>0,95)$ gefunden werden, für die Obstanlage $S$ hingegen trat keine Korrelation zwischen den beiden Parametern auf. Aufgrund dieser Ergebnisse kamen wir zum Schluss, dass die Heterogenität zwischen den Bäumen (,intertree" durch die heterogene Verteilung des $87 \mathrm{Sr} / 86 \mathrm{Sr}$-Isotopenverhältnisses im Boden (direkt neben dem Baum) ausgelöst wird. Diese Heterogenität im Boden könnte auch die Variabilität zwischen den einzelnen Proben desselben Pflanzenorgans („,intrapart“) erklären: die Wurzeln des Apfelbaums sind in unterschiedliche Richtungen im Boden verteilt und absorbieren das bioverfügbare $\mathrm{Sr}$ auch in der Nähe vom Baum bzw. von tieferen Schichten. Wenn sich die Zusammensetzung des Bodens im Umkreis der Wurzeln unterscheidet werden unterschiedliche Isotopenverhältnisse von den Wurzeln aufgenommen, was sich in der Pflanze wiederspiegelt.

Das Sr-Isotopenverhältnis der Bodenproben aus der Obstanlage $S$, entnommen aus unterschiedlichen Schichten (zwischen 10$80 \mathrm{~cm}$ ) variierte sehr stark (Mittelwert \pm Standardabweichung: 0,72142 $\pm 0,00150$ ), wohingegen es bei Obstanlage $L$ relativ konstant blieb (Mittelwert \pm Standardab- 
Tab. 4: Ergebnisse der ${ }^{87} \mathrm{Sr} /{ }^{86} \mathrm{Sr}$-Isotopenverhältnisanalysen der Bodenproben $(10-20 \mathrm{~cm}$ Tiefe), jeder Wert entspricht dem Mittelwert aus den dre Proben, die neben jedem untersuchten Baum gezogen wurden [29]. L=Laimburg, S=Schluderns. // Results of the ${ }^{87} \mathrm{Sr} /{ }^{86} \mathrm{Sr}$ ratio analysis of soil samples collected at 10-20 cm depth. Each value derives from the mean of the three soil samples collected beneath each tree [29]. L=Laimburg, S=Schluderns.

\begin{tabular}{|c|c|c|c|c|c|c|c|c|}
\hline $\begin{array}{c}\text { Obstanlage } \\
\text { orchard }\end{array}$ & & & & & & & $\begin{array}{l}\text { Mittelwert pro Anlage } \\
\text { mean per orchard }\end{array}$ & $\begin{array}{l}\text { Standard- } \\
\text { abweichung } \\
\text { pro Anlage } \\
\text { sd per orchar }\end{array}$ \\
\hline \multirow{3}{*}{ L } & tree & $\mathrm{L} 1 / \mathrm{L} 2$ & L3 & L4 & L5 & L6 & & \\
\hline & mean per tree $(n=3)$ & 0,71078 & 0,70928 & 0,70914 & 0,70968 & 0,71003 & 0,70978 & 0,00066 \\
\hline & sd per tree & 0,00027 & 0,00018 & 0,00053 & 0,00016 & 0,00023 & & 0,00027 \\
\hline \multirow{3}{*}{$\mathrm{S}$} & tree & S1 & S2 & S3 & S4 & S5/S6 & & \\
\hline & mean per tree $(n=3)$ & 0,71546 & 0,715 & 0,71622 & 0,71699 & 0,7156 & 0,71586 & 0,00077 \\
\hline & sd per tree & 0,00094 & 0,00069 & 0,00158 & 0,00032 & 0,00119 & & 0,00094 \\
\hline
\end{tabular}

weichung: 0,71035). Unterschiedliche Faktoren könnten diese inhomogene Verteilung des $87 \mathrm{Sr} / 86 \mathrm{Sr}$-Isotopenverhältnisses in Schluderns beeinflusst haben wie das Klima, die Topographie und die Vegetation. Diese Faktoren beeinflussen und verändern nachweislich die Entstehung des Bodens [16]. Ein Aspekt, der auch nicht unwesentlich dazu beigetragen haben könnte, ist, dass der Boden dieser Obstanlage in der Vergangenheit, mit Erde aus einem anderen Gebiet aufgefüllt wurde. Da dieser Vorgang jedoch schon einige Jahrzehnte zurückliegt, sind nur unzureichende Informationen darüber verfügbar.

\section{SCHLUSSFOLGERUNGEN}

Durch dieses Experiment konnten wir zeigen, dass sich das 87Sr/86Sr-Isotopenverhältnis innerhalb derselben Obstanlage unterscheiden kann, wie das Ergebnis in Anlage $S$ zeigt. Dieser Effekt könnte auch durch das Einbringen von Bodenmaterial in den 30er Jahren verursacht worden sein. In
Anlage $\mathrm{L}$ hingegen zeigte sich, dass das Isotopenverhältnis auch über ein größeres Areal gleichbleiben kann. Kleine Unterschiede zwischen Unterproben derselben Pflanzenorgane eines Baumes (,intrapart“) und Unterschiede zwischen den Bäumen (,intertree“) hängen mit der Variabilität des 87Sr/86Sr-Isotopenverhältnisses im Boden zusammen. Aufgrund der homogenen Verteilung des $87 \mathrm{Sr} / 86 \mathrm{Sr}$ innerhalb eines Baumes (,intratree“) (zwischen Trieben, Blätter, Apfelfruchtfleisch und Apfelschale) sind

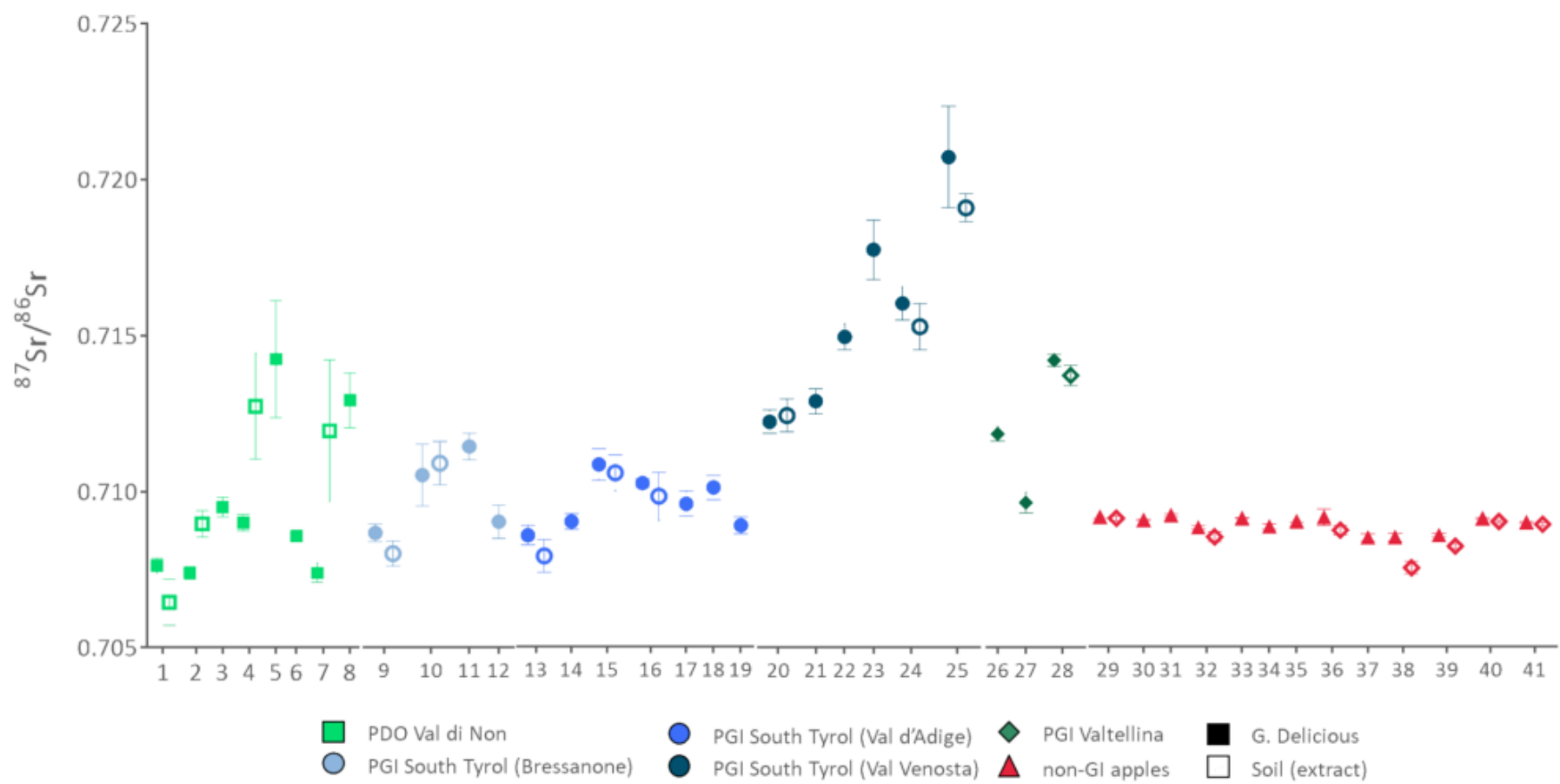

Abb. 6: Verteilung des durchschnittlichen 87Sr/86Sr-Verhältnisses der Äpfel (Golden Delicious) und des Bodens pro Obstanlage unterteilt nach Anbaugebiet. Jeder Punkt steht für den Mittelwert \pm Standardabweichung von 10 Früchten bei Äpfelproben und 5 Proben beim Boden. // Distribution of the mean $87 \mathrm{Sr} / 86 \mathrm{Sr}$ ratio of apples (Golden Delicious) and soil per orchard grouped according to the cultivation district. Each point represents the mean \pm standard deviation of 10 fruits in case of apples and 5 samples in case of soils.

Source: [14], p. 118. 
zukünftige Probennahmen von all diesen Bestandteilen möglich, ohne das Ergebnis negativ zu beeinflussen. Dieser Aspekt ist von großer Bedeutung, da Früchte im Normalfall nur saisonal verfügbar sind. Das $87 \mathrm{Sr} / 86 \mathrm{Sr}$ Isotopenverhältnis variiert aufgrund der lokalen geologischen Bedingungen. Dabei spielt die geologische Komplexität des Bodens eine wichtige Rolle. Das 87Sr/86Sr-Isotopenverhältnis zeigt in geologisch nicht komplexen Gebieten, wie z.B. in alten Ozeanbecken, eine geringe Variabilität wohingegen es in komplexeren Gebieten wie Gebirgsregionen, eine größere Variabilität aufweist. Aufgrund dieser geologischen Unterschiede kann es in kleinen, begrenzten Gebieten zu einer großen Spannbreite an 87Sr/86Sr-Isotopenverhältnissen kommen [24] [47].

\section{EXPERIMENT 3 - AUSWEITUNG DER ANALYSEN AUF 41 STAND- ORTE IN NORDITALIEN}

Das Isotopenverhältnis aller Äpfel die 2017 in 41 Anlagen in Norditalien geerntet wurden reichte von 0,7074 bis 0,7207 [14]. Die drei Hauptanbaugebiete in Südtirol (Unterland, Vinschgau, Brixen Umland) wiesen nicht alle dasselbe Verhältnis auf (Abb. 6), wobei die Äpfel aus Brixen ein sehr ähnliches durchschnittliches Verhältnis wie jene aus dem Etschtal hatten $(0,70983 \pm 0,00130$ und 0,70960 $\pm 0,00083$ ). Die Äpfel aus dem Vinschgau verfügten im Durchschnitt über das höchste $87 \mathrm{Sr} / 86 \mathrm{Sr}$-Verhältnis $(0,7159 \pm$ 0,0032 ), jedoch hatten die Werte der insgesamt sechs Obstanlagen eine große Spannbreite und somit kein über das gesamte Gebiet homogenes Verhältnis. Dies könnte auf die bereits zuvor erwähnte geologische Komplexität in Gebirgsregionen zurückzuführen sein [28]. Die Äpfel aus der Valtellina (Durchschnittliches Isotopenverhältnis $0,71189 \pm 0,00228)$ und Val di Non $(0,70958$ $\pm 0,00261)$ zeigten ebenfalls eine hohe Variabilität und geringe homogene Verteilung, was wiederum, wie im Vinschgau, an der geologischen Komplexität dieser Gebiete liegen kann (Abb. 2). Im Etschtal hingegen verlief die Spannbreite des Isotopenverhältnisses nur innerhalb eines kleinen Bereichs $(0,70857-0,71084)$. Für alle weiteren untersuchten Anbaugebiete ohne geographische Kennzeichnung (non-Gl) konnte eine sehr homogene Verteilung des Verhältnisses aufgezeigt werden, trotz der großen Fläche dieses Anbaugebietes, welches sich über vier verschiedene Regionen Italiens erstreckt (durchschnittlicher Wert: 0,70893 \pm

\section{ZUSAMMENFASSUNG}

Die meisten Konsumenten interessieren sich immer stärker für die Herkunft ihrer Lebensmittel. Eine vielversprechende Methode, um die Herkunft von landwirtschaftlichen Produkten zu prüfen ist die Analyse des Strontium-Isotopenverhältnisses. Der folgende Beitrag zeigt einige Aspekte des 87Sr/86Sr-Verhältnisses und seiner Verwendung als geographischen Indikator für Äpfel auf. In einem ersten Experiment betrachteten wir den Einfluss von landwirtschaftlichen Praktiken auf das Isotopenverhältnis der Apfelbäume im Gewächshaus. Die Bäume passten sich an das Isotopenverhältnis im Boden an. Die landwirtschaftlichen Anwendungen hatten nur einen geringen Einfluss. Im zweiten Experiment wurden Unterschiede im Isotopenverhältnis zwischen Teilproben eines Teiles derselben Pflanze (,intra-part“), zwischen den verschiedenen Bestandteilen desselben Baumes („intra-tree“), zwischen den Bestandteilen unterschiedlicher Bäume derselben Obstanlage (,inter-tree") und ihr Zusammenhang mit dem Isotopenverhältnis im Boden, in jeweils zwei Südtiroler Obstanlagen untersucht. „Intra-part“ und „intra-tree“ war das 87Sr/86Sr Verhältnis homogen, allerdings konnten wir Unterschiede zwischen den Bäumen („,inter-tree“) der beiden Obstanlagen beobachten. Im letzten Experiment wurden die Analysen auf 41 Obstanlagen in Norditalien ausgeweitet. Das Isotopenverhältnis im Apfel korrelierte stark mit jenem des jeweiligen Bodens der entsprechenden Obstanlage. Einige Gebiete hatten ähnliche geologische Eigenschaften und Isotopenverhältnis, wodurch eine eindeutige Unterscheidung nicht für alle Ortschaften möglich war. Trotzdem kann das 87Sr/86SrVerhältnis als geographischer Indikator verwendet werden, jedoch sollten in zukünftigen Studien weitere Parameter miteinbezogen werden.

\section{RIASSUNTO}

L'origine dei prodotti alimentari è sempre più importante per i consumatori. Un metodo promettente per determinare l'origine dei prodotti agricoli è l'analisi del rapporto isotopico dello stronzio (87Sr/86Sr). II presente contributo riporta alcuni aspetti del rapporto $87 \mathrm{Sr} / 86 \mathrm{Sr}$, e l'uso di questo parametro come tracciante geografico per le mele. In un primo esperimento abbiamo studiato l'influenza delle pratiche agricole sul rapporto isotopico dei meli in condizioni controllate in serra. II rapporto isotopico dei meli ha raggiunto il valore del rapporto del suolo. Le pratiche agricole hanno influenzato solo modestamente. In un secondo esperimento abbiamo studiato il rapporto $87 \mathrm{Sr} / 86 \mathrm{Sr}$ nei meli in termini di variabilità intra-parte (differenze tra sotto-campioni dello stesso organo in un albero), intra-albero (differenze tra i organi dello stesso albero) e inter-albero (differenze tra ogni organo di alberi diversi nello stesso campo) ed in confronto con il rapporto isotopico del suolo corrispondente. In entrambi i meleti, il rapporto sia intraparte che intra-albero era piuttosto omogeneo, ma considerando la variabilità inter-albero sono emerse differenze tra i due meleti. Una terza indagine ha riguardato l'analisi del rapporto $87 \mathrm{Sr} / 86 \mathrm{Sr}$ in 41 campi, distribuiti nel Nord Italia. II rapporto isotopico era altamente correlato con quello del suolo corrispondente. Aree di coltivazione con caratteristiche geologiche simili sono caratterizzate da rapporti $87 \mathrm{Sr} / 86 \mathrm{Sr}$ simili, e quindi una completa separazione delle aree non è stata possibile. Questi risultati confermano il potenziale del rapporto $87 \mathrm{Sr} / 86 \mathrm{Sr}$ come tracciante geografico, ma studi futuri dovrebbero includere altri parametri. 
0,00024). Die 87Sr/86Sr-Verhältnisse der Äpfel wurden im Jahre 2018 mit jenen aus dem dazugehörigen Boden verglichen und es konnte eine starke Korrelation $(R>0,98)$ zwischen den Apfelproben und den dazugehörigen Böden, gefunden werden. Dieses Ergebnis stimmt mit einigen aus der Literatur überein [47] [48] [49].

Auf Grundlage dieser Ergebnisse war es nicht möglich, geographisch gekennzeichnete (geschützte geographische Angabe und geschützte Ursprungsbezeichnung) und nicht-gekennzeichnete Äpfel aus Norditalien zu unterscheiden. Zwar unterschieden sich einige der Verhältnisse signifikant voneinander, wie jene aus dem Vinschgau von jenen aus dem Etschtal, Val di Non und den nicht gekennzeichneten Äpfeln, jedoch überlappten sich die Verhältnisse aus der Valtellina und Brixen signifikant mit jenen von all den anderen untersuchten Gebieten (Abb. 6). In diesem Fall war es aufgrund der ähnlichen geologischen Merkmale dieser Anlagen nicht möglich sie eindeutig voneinander zu unterscheiden.

2018 wurden aus 20 der 41 bereits 2017 untersuchten Obstanlagen erneut Apfelproben entnommen und analysiert. Das Isotopenverhältnis reichte von 0,7071-0,7212. Die beiden Datensätze von 2017 und 2018 wurden verglichen und es zeigte sich eine sehr hohe Korrelation $(R>0,996, p<0,01)$ zwischen den beiden Jahren. In manchen Fällen stimmten die Daten von 2018 sogar exakt mit jenen von 2017 überein. Diese Ergebnisse bestätigen, dass das $87 \mathrm{Sr} / 86 \mathrm{Sr}$-Verhältnis ein stabiler Indikator ist, der auch nach Ablauf einer bestimmten Zeit (in diesem Fall ein Jahr) nicht variiert [50].

\section{SCHLUSSFOLGERUNGEN}

In dieser Studie wurde untersucht, ob das 87Sr/86Sr-Isotopenverhältnis geeignet ist, um Äpfel aus verschiedenen Anbaugebieten zu unterscheiden. Das Isotopenverhältnis des Apfels korreliert sehr stark mit jenem aus dem dazugehörigen Boden. Somit kann durch diese Studie bestätigt werden, dass das $87 \mathrm{Sr} / 86 \mathrm{Sr}$-Verhältnis ein hilfreicher Indikator ist, um den geographischen Ursprung von Äpfeln zu ermitteln. Da jedoch der Boden an unterschiedlichen Standorten über eine ähnliche Sr-Isotopenzusammensetzung verfügen kann (wie zum Beispiel im Südtiroler Unterland und in der Poebene) ist eine alleinige Unterscheidung nur auf Basis des 87Sr/86Sr-Verhältnisses nicht immer möglich. Um die Herkunft von Äpfeln aus allen Anbaugebieten eindeutig nachzuweisen sind weitere Studien notwendig, die weitere Parameter, welche Aussagen über den geographischen Ursprung ermöglichen, wie z.B. eine Multielement-Analyse oder das Isotopenverhältnis von leichteren Elementen wie $\mathrm{C}, \mathrm{H}, \mathrm{O}$ und $\mathrm{N}$, in die Untersuchungen miteinbeziehen. Es konnte jedoch gezeigt werden, dass bei unterschiedlicher geologischer $\mathrm{Zu}$ sammensetzung der Böden eine Unterscheidung der Äpfel und eine entsprechende Zuordnung zu verschiedenen Anbaugebieten sehr wohl möglich ist. Bestimmte Anbaugebiete wie das Vinschgau heben sich durch ein hohes Strontium Isotopenverhältnis von den anderen Apfelanbaugebieten ab. Besonders hervorzuheben ist die sehr gute Korrelation zwischen den Böden und den verschiedenen Pflanzenteilen wie Trieben, Blättern und Äpfeln selbst, sowie auch das Beibehalten des Sr-Isotopenverhältnisses über einen mehrjährigen Zeitraum am sel ben Standort, wodurch die Sr-Analyse eine verlässliche Aussage ermöglicht. Erste Versuche zur Zuordnung von verschiedenen Apfel-Anlieferungen aus unterschiedlichen Ortschaften in diversen Südtiroler Obstmagazinen wurden bereits durchgeführt und konnten vielversprechende Ergebnisse zur Nachverfolgbarkeit der Äpfel auf die einzelne Obstwiesen bringen. Die Zusammenarbeit mit den Südtiroler Obstvermarktungsbetrieben und Verbänden wird laufend intensiviert und ausgebaut.

\section{DANKSAGUNG}

Wir möchten uns bei folgenden Personen und Instituten für ihre Unterstützung bedanken: Lorenzo Berra von Agrion, Luigi Schiavon von Azienda Sasse Rami (Veneto Agricoltura), Luis Clementi von Gebr. Clementi $\mathrm{GmbH}$, Luca Gambarotto von Soc. Agr. Tagliani Vivai International, Luca Folini von der Fondazione Fojanini, Luigi Manfrini von der Universität Bologna, Loris Marchel und allen Technikern von APOT, Wolfgang Graiss von Vi.P Gen. landw. Gesellschaft, Meinrad Zöschg und den Technikern des Südtiroler Beratungsrings, Stephan Raffl, Markus Schöpf, Freddy Facchinelli, Martin Platter und Thomas Linger von der Agentur Landesdomäne, Aldo Matteazzi, Martin Höller, Karin Gummerer und Cristina Gadotti vom Versuchszentrum Laimburg und allen Landwirten, welche uns die Probennahme in ihren Anlagen ermöglicht haben, sowie der Autonomen Provinz Bozen für die finanzielle Unterstützung (Leistungsvereinbarung Umweltwissenschaften-Isotopenanalysen, Beschluss Landesregierung Nr. 1472 vom 07.10.2013 und Projekt TIOMI, Beschluss Landesregierung Nr. 865 vom 04.09.2018). 


\section{LITERATUR}

[1] TNS Opinion \& Social at the request of the European Commission, Directorate-Genera for Agriculture and Rural Development (2012). Europeans' attitudes towards food security, food quality and the countryside Report. (Special Eurobarometer ; 389 / Wave EB77.2). Retrieved April 5, 2020, from https://ec.europa.eu/commfrontoffice/pub licopinion/archives/ebs/ebs 389 en.pdf.

[2] Krystallis, A. (2017) The concept of authenticity and its relevance to consumers: Country and place branding in the context of food authenticity. In: Constantinos A.G. Georgios P.D. (eds.). Food Authentication. Management, Analysis and Regulation. John Wiley \& Sons, Chichester, United Kingdom, pp 42-82.

[3] Kelly S., Heaton K., Hoogewerff J. (2005) Tracing the geographical origin of food: The application of multi-element and multi-isotope analysis. Trends in Food Science and Technology 16 (12), 555-567, DOI: $10.1016 /$ j.tifs.2005.08.008.

[4] Bérard L., Marchenay P. (2006). Local products and geographical indications. Taking ac count of local knowledge and biodiversity International Social Science Journal 58 (187), 109-116, DOI: 10.1111/i.14682451.2006.00592.x.

[5] Deselnicu O.C., Costanigro M., Souza-Monteiro D.M. (2013). A meta-analysis of geographical indication food valuation studies. What drives the premium for origin-based labels? Journal of Agricultural and Resource Economics 38 (2), 204-219, DOI: 10.22004/ag.econ.158285.

[6] Ehleringer J. R., Matheson Jr. S. M. (2010) Stable isotopes and courts. Utah Law Review 2010 (2), 385-442. Retrieved April 5, 2020, from https://heinonline.org/HOL/Page?collection=journals\&handle=hein.journals/utahlr2010\&id=390\&men tab=srchresults.

[7] Luykx D.M.A.M., van Ruth S.M. (2008). An overview of analytical methods for determining the geographical origin of food products. Food Chemistry 107 (2), 897-911, DOI: 10.1016/j.foodchem.2007.09.038.

[8] Kelly S., Heaton K., Hoogewerff J. (2005). Tracing the geographical origin of food. The application of multi-element and multi-isotope analysis. Trends in Food Science and Technology 16 (12), 555-567, DOI: 10.1016/j.tifs.2005.08.008.

[9] Drivelos, S.A., Georgiou, C.A. (2012). Multielement and multi-isotope-ratio analysis to determine the geographical origin of foods in the European Union. TrAC Trends in
Analytical Chemistry, 40, 38-51, DOI: 10.1016/j.trac.2012.08.003.

[10] Veizer J. (1989). Strontium isotopes in seawater through time. Annual Review of Earth and Planetary Sciences 17:141-67, DOI: 10.1146/annurev.ea.17.050189.001041.

[11] Banner J.L. (2004). Radiogenic isotopes. Systematics and applications to earth surface processes and chemical stratigraphy. EarthScience Reviews 65 (3-4), 141-194, DOI: 10.1016/S0012-8252(03)00086-2.

[12] Jacobsen S.B., Dymek R.F. (1988). Nd and Sr isotope systematics of clastic metasediments from Isua, West Greenland. Identification of pre-3.8 Ga Differentiated Crustal Components. Journal of Geophysical Research: Solid Earth 93 (B1), 338-354, DOI: 10.1029/JB093iB01p00338.

[13] Bentley R.A. (2006). Strontium isotopes from the earth to the archaeological skeleton. A review. Journal of Archaeological Method and Theory 13, 135-187, DOI: 10.1007/s10816-006-9009-x.

[14] Aguzzoni A. (2018). 87Sr/86Sr ratio of apples and its use as geographical tracer. PhD Dissertation, Freie Universität Bozen. Bozen, Italien.

[15] Drouet T., Herbauts J., Gruber W. et al. (2007). Natural strontium isotope composition as a tracer of weathering patterns and of exchangeable calcium sources in acid leached soils developed on loess of central Belgium. European Journal of Soil Science 58 (1), 302-319, DOI: 10.1111/j.13652389.2006.00840.x.

[16] Crowley B.E., Miller J.H., Bataille C.P. (2017). Strontium isotopes $(87 \mathrm{Sr} / 86 \mathrm{Sr})$ in terrestrial ecological and palaeoecological research. Empirical efforts and recent advances in continental-scale models. Biological Reviews 92 (1), 43-59, DOI: 10.1111/brv.12217.

[17] Kabata-Pendias A. (2011). Trace elements in soils and plants. CRC Press/Taylor \& Francis Group, Boca Raton, Florida, USA, hier p. 548

[18] Lyubomirova V., Mihaylova V., Djingova R. (2015). Effects of soil properties and anthropogenic activity on the transfer of 52 elements in the system soil/Taraxacum officinale. Journal of Soils and Sediments 15 1549-1557, DOI: 10.1007/s11368-015-1094$\underline{6}$.

[19] Wang C.J., Wang J.J., Chiu C.Y. et al. (2000). Transfer factors of $90 \mathrm{Sr}$ and $137 \mathrm{Cs}$ from soil to the sweet potato collected in Taiwan. Journal of Environmental Radioactivity 47 (1), 15-27, DOI: 10.1016/S0265931X(99)00013-2.
[20] Horsky M., Irrgeher J., Prohaska T. (2016). Evaluation strategies and uncertainty calculation of isotope amount ratios measured by MC ICP-MS on the example of Sr. Analytical and Bioanalytical Chemistry 408 (2), 351367, DOI: 10.1007/s00216-015-9003-9.

[21] Yang L. (2009). Accurate and precise determination of isotopic ratios by MC-ICP-MS. A review. Mass spectrometry Reviews 28 (6), 990-1011, DOI: 10.1002/mas.20251.

[22] Horn P., Schaaf P., Holbach B. et al. (1993). $87 \mathrm{Sr} / 86 \mathrm{Sr}$ from rock and soil into vine and wine. Zeitschrift für Lebensmittel-Untersuchung und Forschung 196 (5), 407-409, DOI: 10.1007/BF01190802.

[23] Flockhart D.T., Kyser T.K., Chipley D. et al. (2015). Experimental evidence shows no fractionation of strontium isotopes (87Sr/86Sr) among soil, plants, and herbivores. Implications for tracking wildlife and forensic science. Isotopes in Environmental and Health Studies 51 (3), 372-381, DOI: 10.1080/10256016.2015.1021345.

[24] Blum J.D., Taliaferro E.H., Weisse M.T. Holmes R.T. (2000). Changes in Sr/Ca, Ba/Ca and $87 \mathrm{Sr} / 86 \mathrm{Sr}$ ratios between trophic levels in two forest ecosystems in the northeastern USA. Biogeochemistry 49 (1), 87-101, DOI: 10.1023/A:1006390707989.

[25] Green G.P., Bestland E.A., Walker G.S (2004). Distinguishing sources of base cations in irrigated and natural soils. Evidence from strontium isotopes. Biogeochemistry 68 (2), 199-225, DOI: 10.1023/B:BIOG.0000025743.34079.d3.

[26] Techer I., Medini S., Janin M. et al. (2017). Impact of agricultural practice on the $\mathrm{Sr}$ isotopic composition of food products: Application to discriminate the geographic origin of olives and olive oil. Applied Geochemistry 82, 1-14, DOI: 10.1016/j.apgeochem.2017.05.010.

[27] Techer I., Lancelot J., Descroix F. et al. (2011). About Sr isotopes in coffee 'Bourbon Pointu' of the Réunion Island. Food Chemistry, 126 (2), 718-724, DOI: 10.1016/j.foodchem.2010.11.035.

[28] Aguzzoni A., Bassi M., Robatscher P. et al. (2018). Plant Sr isotope ratios as affected by the $\mathrm{Sr}$ isotope ratio of the soil and of the external Sr inputs. Journal of Agricultural and Food Chemistry 66 (40), 10513-10521, DOI: 10.1021/acs.jafc.8b02604.

[29] Aguzzoni A., Bassi M., Robatscher P. et al. (2019). Intra-and Intertree Variability of the 87Sr/86Sr Ratio in Apple Orchards and Its Correlation with the Soil 87Sr/86Sr Ratio. Journal of Agricultural and Food Chemistry 67 (20), 5728-5735, DOI: $10.1021 /$ acs.jafc.9b01082. 
[30] Agrios - Arbeitsgruppe für integrierten Obstbau in Südtirol (ed.) (2020). Richtlinien für den Integrierten Kernobstbau 2020. Retrieved March 20,2020, from http://www.agrios.it/fuer-obstbauern/richtlinien-fuer-denintegrierten-kernobstbau-2020/.

[31] Istituto Superiore per la Protezione e la Ricerca Ambientale (ISPRA) (n. d.). Carta Geologica d'Italia Scala 1:100 000. Retrieved March 20, 2020, from http://193.206.192.231/carta geologica italia/default.htm.

[32] Durante C., Baschieri C., Bertacchini L. et al. (2013). Geographical traceability based on 87Sr/86Sr indicator. A first approach for PDO Lambrusco wines from Modena. Food Chemistry 141 (3), 2779-2787, DOI: 10.1016/j.foodchem.2013.05.108.

[33] Aguzzoni A., Bassi M., Pignotti E. et al. (2020). Sr isotope composition of Golden Delicious apples in Northern Italy reflects soil $87 \mathrm{Sr} / 86 \mathrm{Sr}$ ratio of the cultivation area. Journal of the Science of Food and Agriculture 100 (9), DOI: 10.1002/jsfa.10399.

[34] Technical Committee ISO/TC 190 Subcommittee SC3 (ed.) (2008). Soil Quality - Extraction of Trace Elements from Soil Using Ammonium Nitrate Solution. ISO-Norm ISO 19730:2008. International Organization for Standardization (ISO), Genf, Schweiz.

[35] Swoboda S., Brunner M., Boulyga S.F. et al. (2008). Identification of Marchfeld asparagus using $\mathrm{Sr}$ isotope ratio measurements by MC-ICP-M. Analytical and Bioanalytical Chemistry 390 (2), 487-494, DOI: 10.1007/s00216-007-1582-7.

[36] Lagad R.A., Singh S.K., Rai V.K. (2017). Rare earth elements and $87 \mathrm{Sr} / 86 \mathrm{Sr}$ isotopic characterization of Indian Basmati rice as potential tool for its geographical authenticity. Food Chemistry 217, 254-265, DOI: 10.1016/i.foodchem.2016.08.094.
[37] Hiraoka H., Morita S., Izawa A. et al. (2016). Tracing the geographical origin of onions by strontium isotope ratio and strontium content. Analytical Sciences 32 (7), 781-788, DOI: 10.2116/analsci.32.781.

[38] Meija J., Coplen T.B., Berglund M. et al. (2016). Isotopic compositions of the elements 2013 (IUPAC technical Report). Pure and Applied Chemistry 88 (3), 293-306, DOI: 10.1515/pac-2015-0503.

[39] Granato D., de Araújo Calado V.M., Jarvis B. (2014). Observations on the use of statistical methods in food science and technology. Food Research International 55, 137-149, DOI: 10.1016/j.foodres.2013.10.024.

[40] Mercurio M., Grilli E., Odierna P. et al. (2014). A 'Geo-Pedo-Fingerprint'(GPF) as a tracer to detect univocal parent material-towine production chain in high quality vineyard districts, Campi Flegrei (Southern Italy). Geoderma 230, 64-78, DOI: 10.1016/j.geoderma.2014.04.006.

[41] West J.B., Hurley J.M., Dudás F.Ö. et al. (2009). The stable isotope ratios of marijuana. II. Strontium isotopes relate to geographic origin. Journal of Forensic Sciences 54 (6), 1261-1269, DOI: 10.1111/j.15564029.2009.01171.x.

[42] Schmitt A.D., Gangloff S., Labolle F. et al. (2017). Calcium biogeochemical cycle at the beech tree-soil solution interface from the Strengbach CZO (NE France). Insights from stable $\mathrm{Ca}$ and radiogenic $\mathrm{Sr}$ isotopes. Geochimica et Cosmochimica Acta 213, 91-109, DOI: 10.1016/j.gca.2017.06.039.

[43] Pandey R. (2015). Mineral nutrition of plants. In: Bahadur B., Venkat Rajam M., Sahijram L. (eds.). Plant biology and biotechnology. Springer, New Delhi, India, pp. 499538, DOI: 10.1007/978-81-322-2286-6 20.
[44] van der Heijden G., Dambrine E., Pollier B. et al. (2015). Mg and Ca uptake by roots in relation to depth and allocation to aboveground tissues. Results from an isotopic labeling study in a beech forest on base-poor soil. Biogeochemistry 122 (2-3), 375-393, DOI: $10.1007 / \mathrm{s} 10533-014-0047-2$.

[45] Tchaikovsky A., Irrgeher J., Zitek A. et al. (2017). Isotope pattern deconvolution of different sources of stable strontium isotopes in natural systems. Journal of Analytical Atomic Spectrometry 32 (11), 23002307, DOI: 10.1039/C7JA00251C.

[46] Rummel S., Hoelzl S., Horn P. et al. (2010) The combination of stable isotope abundance ratios of $\mathrm{H}, \mathrm{C}, \mathrm{N}$ and $\mathrm{S}$ with $87 \mathrm{Sr} / 86 \mathrm{Sr}$ for geographical origin assignment of orange juices. Food Chemistry 118 (4), 890900, DOI: 10.1016/j.foodchem.2008.05.115.

[47] Aoyama K., Nakano T., Shin K.-C. (2017). Variation of strontium stable isotope ratios and origins of strontium in Japanese vegetables and comparison with Chinese vegetables. Food Chemistry 237, 1186-1195, DOI: 10.1016/j.foodchem.2017.06.027.

[48] Durante C., Bertacchini L., Cocchi M. et al. (2018). Development of $87 \mathrm{Sr} / 86 \mathrm{Sr}$ maps as targeted strategy to support wine quality. Food Chemistry 255, 139-146 DOI: 10.1016/j.foodchem.2018.02.084.

[49] Tescione I., Marchionni S., Casalini M. et al. (2018). 87Sr/86Sr isotopes in grapes of different cultivars. A geochemical tool for geographic traceability of agriculture products. Food Chemistry 258, 374-380 DOI: 10.1016/j.foodchem.2018.03.083.

[50] Durante C., Baschieri C., Bertacchini L. et al. (2015). An analytical approach to $\mathrm{Sr}$ isotope ratio determination in Lambrusco wines for geographical traceability purposes. Food Chemistry 173, 557-563, DOI: 10.1016/i.foodchem.2014.10.086.

\section{(2) $(1) \Theta$}

Dieses Werk ist lizenziert unter einer Creative Commons Namensnennung-Nicht kommerziell 4.0 International Lizenz.

Quest'opera è distribuita con Licenza Creative Commons Attribuzione - Non commerciale 4.0 Internazionale.

This work is licensed under a Creative Commons Attribution-NonCommercial 4.0 International License.

Für alle Abbildungen und Tabellen ohne Nennung des Urhebers gilt: (C) Versuchszentrum Laimburg.

Per tutte le immagini e tabelle senza menzione dell'artefice vale: (C) Centro di Sperimentazione Laimburg.

For all figures and tables without mention of the originator applies: (C) Laimburg Research Centre. 